\title{
Retention of Capable New Employees under Uncertainty: Impact of Strategic Interactions
}

\author{
H. Dharma Kwon \\ Department of Business Administration, University of Illinois at Urbana-Champaign, \\ Champaign, Illinois 61820, USA, dhkwon@illinois.edu \\ Onesun Steve Yoo \\ UCL School of Management, University College London, \\ 1 Canada Square, London E14 5AB, United Kingdom, onesun.yoo@ucl.ac.uk
}

\begin{abstract}
We study a game involving a firm and a newly hired employee whose capability is initially unknown to both parties. Both players observe the performance of the employee and update their common posterior beliefs about the employee's capability. The learning process presents each party with an option: the firm can terminate an incapable employee, and a capable employee can leave the firm for greater financial remuneration elsewhere. To understand the impact of this noncooperative interaction, we examine the Markov perfect equilibrium termination strategies and payoffs that unfold. We find that in the region of sufficiently high learning rates, reducing the rate of learning can increase the equilibrium payoff for both parties. Slower learning prolongs the employment because more performance outcomes must be observed for assessing the employee's capability. In the region of sufficiently slow learning rates, reducing the rate of learning can benefit the firm if the employee is deemed capable but hurt the firm otherwise. Our result identifies a nonfinancial way for firms to improve retention of capable new employees.
\end{abstract}

Key words: Bayesian sequential decision; employee retention; timing game; stochastic dynamic game

\section{Introduction}

A newly hired or promoted employee faces a set of challenges arising from lack of experience and uncertainty regarding productivity in the new role. Hence, the likelihood of the employee's success in the new role is initially unknown to both the firm and the employee. As the employee compiles a track record, both parties concurrently learn about the employee's capabilities. This learning process presents each party with an option: The firm can terminate an incapable employee, and a 
capable employee can voluntarily leave the firm for greater financial remuneration elsewhere. The following examples typify the context of the firm-employee relationship that we examine in this study.

- Promotion into a new role. An associate in a consulting firm is promoted to the position of engagement manager responsible for attracting new clients, for which the employee has limited experience. Hence, despite their shared optimism based on the employee's track record in the previous role, both the firm and the employee are initially uncertain about whether the employee will succeed in the new role. They will find this out together as they both observe the revenue the employee generates for the firm. The firm will terminate an unproductive engagement manager, while a proficient employee might well move to a competing firm that can provide greater financial remuneration.

- Hiring into a different environment. A successful executive from an established firm joins a start-up company as a vice president (VP), to run its day-to-day operations. Given the unfamiliar and unpredictable circumstances of small entrepreneurial firms, both the firm and the new VP are initially wary about whether the VP's past success will translate successfully into the new environment. They will learn this together by observing sales trends. The firm will replace a VP who is not capable of achieving certain milestones, and a successful VP may choose to join a different venture with greater potential and financial remuneration.

- Starting a new career. A recent MBA graduate is hired by a company. Despite the MBA's promise, neither party knows whether the MBA will be capable in the new managerial role. Both parties will learn about the new employee's capabilities after observing the employee's performance over time. The company will terminate an incompetent employee, while a successful employee can move to a different company in response to a better financial offer.

While firms and employees desire cooperative work relationships that stimulate both parties to act on behalf of their mutual benefit, this is often not the case in practice. As the uncertainty about the employee's capability resolves during the employment relationship, the presence of these exercisable options can prompt the two parties to behave noncooperatively (leading to an uneasy atmosphere in the workplace). For firms that frequently hire or promote employees, it is important to understand how the noncooperative interactions influence the employment dynamics and the firm's ability to retain its capable employees.

In this paper, we investigate the role of Bayesian learning about employee capability and examine whether firms should encourage (or empower) their newly hired or promoted employees to engage in 
tasks for which luck plays a relatively small/large role in performance outcomes. From an intuitive perspective, a higher learning rate, i.e., being able to learn about the employee's capability more quickly, may appear to be desirable for both parties. However, in a noncooperative setting, the firm can exploit the higher learning rate to undermine the employee and vice versa, so it is unclear which player benefits more from an increased learning rate. Thus, the central question of this paper is: Which player benefits from a higher rate of learning?

To examine this question, we model the noncooperative interaction between the firm and the employee as a stopping time game, obtain the equilibrium strategies and payoffs, and analyze the impact of the learning rate. The employee is assumed to be either capable (high capability type) or not capable (low capability type). Initially, the employee's capability type is unknown to both the firm and the employee, and they share a common prior probability that the employee is of the high type. As the employee contributes to the firm's profit stream over time, both the firm and the employee observe this public information and continuously update their beliefs in a Bayesian fashion; thus, if high (respectively, low) performance is observed, then this belief will increase (respectively, decrease). Although the updating of their beliefs may in practice evolve differently for the firm and the employee, we assume that there is minimal disagreement in their conceptions regarding the same information, i.e., as per the argument made by Aumann (1976), after a particular performance is observed, it is not possible for one party to think the employee is of the high capability type while the other thinks the employee is of the low capability type. We therefore model the evolution of their beliefs in an identical manner. Such an assumption is also made by others; see, e.g., Papageorgiou (2013).

At any time during the learning process, either player can terminate the employment relationship: The firm can dismiss an employee who is deemed to be of low capability, and an employee who learns of his or her high capability can quit in favor of higher compensation elsewhere. After the employment relationship is terminated, the firm anticipates a profit stream without the employee and the employee anticipates an outside option that reflects the employee's true capabilities. Both 
parties can initiate separation based on their posterior belief about the employee's capabilities. In the unique Pareto-dominant Markov perfect equilibrium (MPE) that we obtain, if this posterior belief falls below a lower threshold, the firm terminates the employee, whereas if it exceeds an upper threshold, the employee leaves the firm.

We analyze the effect of the learning rate on the equilibrium strategies and payoffs and compare it to a benchmark, the cooperative Nash bargaining payoffs. In the cooperative model, both parties (employee and the employer) work together to optimize the total payoff and then split the surplus total payoff (net of the outside options) according to the Nash bargaining solution (Nash 1950). That means that the employee and the employer terminate their relationship at the optimal time to maximize the sum total of the payoffs, which is then divided between the two by a pre-determined formula that was agreed upon. In the Nash bargaining solution, we find that a higher learning rate always benefits both parties. In contrast, the effect of the learning rate is more nuanced in the noncooperative setting. In particular, in the region of sufficiently high learning rates, the opposite can be true: faster learning can decrease the payoffs for both the firm and the employee at the same time. In other words, in the noncooperative setting between the firm and the employee, slower learning can simultaneously benefit both parties. Slower learning prolongs the employment relationship because more performance outcomes must be observed in order to evaluate the employee. Since both parties retain the option of discontinuing the employment relationship at any time, both can minimize their downside risks and take advantage of the relationship for a longer period: the firm can retain a capable employee longer while minimizing the downside risk of paying an incapable employee, and at the same time, an incapable employee can remain with the firm longer and still maintain his or her outside option. We show numerically that this counterintuitive result is robust to potential wage changes for rewarding high performance as well as to on-the-job learning that transforms low capability type employees to high capability type employees.

In addition, we identify which player benefits from a higher learning rate based on the learning rate and the shared belief. Our results thus point to active nonfinancial strategies that firms can 
employ to increase the retention of capable new employees. Firms can control the rate of learning by assigning (or encouraging/empowering) their newly hired/promoted employees to take on tasks for which their capability will be revealed quickly or slowly (i.e., depending on whether luck plays a relatively small/large role in the performance). Depending on how its belief about the capability evolves, the firm can manage the learning rate appropriately.

From the perspective of game theory, this paper contributes to the literature that examines how information acquisition (or learning) impacts the equilibrium payoff of the players. Specifically, we present a surprising example in which slower acquisition of information may improve the payoffs of both players. While there exist well-known examples of games with incomplete and asymmetric information in which acquisition of additional information hurts all players (see, for example, Kreps 1988, p. 41), our model presents an example of an incomplete information game in which a similar result holds despite the information being symmetric.

The Bayesian framework that we utilize is based on the work of Shiryaev (1967), who studied the single-player decision-theoretic problem of minimizing the cost of errors under two hypotheses about the drift of a one-dimensional Brownian motion. This same framework has been applied in various decision-theoretic settings - for example, by Ryan and Lippman (2003), who considered when to abandon a project of unknown profitability, by Decamps et al. (2005), who identified the optimal time to invest in an asset of unknown underlying value, and by Kwon (2010), who studied an expansion and exit decision regarding a pilot project of unknown profitability (that could be in one of two states). The framework has also been applied to dynamic game models under incomplete information. For example, Bolton and Harris (1999) studied the free-rider problem arising from information externality when many agents face the same uncertainty and experimentation, and Bergemann and Valimaki (2000) examined a multi-agent learning model with two sellers who compete on price and many buyers who experiment with a new product of unknown quality. Unlike these papers, we apply the framework to a game between two non-rivalrous players in an employment relationship. 
We note that the effect of uncertainty has been of particular interest in the economics literature related to decisions concerning real options. In a model of a firm with an option to enter or exit an industry, Dixit (1989) obtains the comparative statics of the optimal entry and exit thresholds with respect to the profit stream's volatility. Alvarez (2003) proved a general comparative statics result for the optimal policy and the optimal return with respect to the level of uncertainty for a class of optimal stopping problems that often arise in economic decisions. The value function generally increases with uncertainty under conventional situations with real options (Dixit 1992). Thus, our key finding also provides a stark contrast to the conventional results of real options models under incomplete information, and this is due to the noncooperative interaction between firm and employee.

Our paper contributes to the literature in labor economics that examines the impact of uncertainty regarding the type of the employee. To explain the high job mobility of inexperienced and young workers, Johnson (1978) formulated a job shopping model in which a worker sequentially tries out jobs to learn about his/her own capability and type. In a similar vein, Viscusi (1980) studied a model of a Bayesian worker who learns about a job's attributes on the job and found that such a worker will prefer jobs with uncertain prospects. Jovanovic (1979) studied a long-run equilibrium theory of turnover in which a worker's match with a job is gradually revealed on the job. Freeman (1977) investigated the employer's wage strategy in response to the performance of the worker, using a two-period model in which the information on a worker's productivity is symmetrically or asymmetrically available to both the employer and the worker. More recent studies include Papageorgiou (2013), who studied the labor market from the perspective of a worker's search for a job that matches his/her skills through changing jobs. The key aim of these studies is to understand how uncertainty impacts labor market dynamics. Our paper complements this line of research by focusing on the firm level dynamics and how the speed of the resolution of uncertainty impacts the retention of newly hired/promoted employees within a firm.

Managing the workforce has also long been an important operational problem. In traditional models, employees have had little leverage over the firm, and the firm unilaterally determines 
whether to retain or terminate its employees (so as to minimize the associated costs). An early stream of literature examined the setting of employees in production plants and proposed mathematical programming models of workforce planning to help firms minimize the long-term costs associated with hiring, overtime pay, and idle time (Holt et al. 1960, Bitran et al. 1981). The literature has also utilized queuing-based staffing models in service contexts to minimize personnel costs and abandonment penalties for call centers (Pinker and Shumsky 2000, Gans and Zhou 2002). Bassamboo et al. (2006) proposed refinements that account for specialization or learning, and Arlotto et al. (2014) examined a similar problem using the multi-armed bandit model with Bayesian learning. In our paper, in contrast, the focal employee directly influences firm profits and thus holds more leverage over the firm. The firm must take the employee's option of voluntarily quitting into account when making a termination decision. On the other hand, recent streams of this research have taken into consideration the strategic behavior of workers for managing their self-scheduling capacity in service systems (e.g., Gurvich et al. 2015). Such endeavors require examining the intrafirm equilibrium dynamics between the firm and its employees. Our work therefore complements these recent lines of research.

The rest of this paper is organized as follows. In Section 2, we formally introduce the model and characterize the payoffs for the firm and the employee. In Section 3, we examine the cooperative central decision model as a benchmark for investigating the impact of Bayesian learning. In Section 4, we resume our study of the noncooperative model to characterize the unique equilibrium strategies and payoffs and examine the effect of learning rates. In Section 5, we examine the robustness of our findings. In Section 6, we discuss some managerial insights, and we conclude in Section 7. All proofs are provided in the Appendix.

\section{Model}

We represent the cumulative profit contributed by the employee as a stochastic process $X=\left\{X_{t}\right.$ : $t \geq 0\}$ given by the Brownian motion

$$
X_{t}=\mu t+\sigma B_{t},
$$


where $\mu$ is the drift (the expected profit per time unit earned by the employee), $\sigma$ is the constant volatility, and $B \equiv\left\{B_{t}: t \geq 0\right\}$ is a Wiener process. The magnitude of the drift $\mu$ represents the employee's capability. The value of $\mu$ is unknown to both the firm and the employee, but it is commonly known to be either $h$ for an employee who is capable (high capability type) or $\ell$ for an employee who is incapable (low capability type), where $h>\ell$. Note that the constant volatility $\sigma$ is independent of the employee's capability, as it reflects the nature of the job or task at hand; thus $\sigma$ represents the degree to which luck contributes to the profit stream. Let $(\Omega, \mathcal{G}, P)$ be the probability space on which $X_{t}, \mu$, and $B_{t}$ are measurable, and let $\mathcal{F}=\left\{\mathcal{F}_{t}: t \geq 0\right\}$ denote the filtration generated by the observable cumulative profit process $X=\left\{X_{t}: t \geq 0\right\}$.

Both the firm and the employee have a common prior $p_{0} \equiv P\left(\{\mu=h\} \mid \mathcal{F}_{0}\right)$, the initial probability (belief) that the employee is capable. We can use the Bayes rule (see Peskir and Shiryaev 2006, pp. 288-289) to derive the following expression for $P_{t} \equiv P\left(\{\mu=h\} \mid \mathcal{F}_{t}\right)$ in terms of the observable process $X_{t}$ :

$$
P_{t}=\left(1+\frac{1-p_{0}}{p_{0}} \exp \left\{-\frac{h-\ell}{\sigma^{2}}\left[X_{t}-\frac{h+\ell}{2} t\right]\right\}\right)^{-1} .
$$

The time evolution (stochastic differential equation) for $P_{t}$ is given by

$$
d P_{t}=\frac{h-\ell}{\sigma} P_{t}\left(1-P_{t}\right) d \tilde{B}_{t}
$$

where

$$
\tilde{B}_{t} \equiv \frac{1}{\sigma}\left(X_{t}-\int_{0}^{t} E\left[\mu \mid \mathcal{F}_{s}\right] d s\right)=\frac{1}{\sigma}\left[X_{t}-\int_{0}^{t}\left(P_{s} h+\left(1-P_{s}\right) \ell\right) d s\right]
$$

is a Wiener process constructed solely from the observable process $X_{t}$ (Liptser and Shiryayev 1977). Both players concurrently observe $X_{t}$ and update their belief about the employee's capability to obtain the common posterior probability $P_{t}$.

Note that the speed of the Bayesian updating is proportional to $\left(\frac{h-\ell}{\sigma}\right) P_{t}\left(1-P_{t}\right)$. In fact, the ratio $\frac{h-\ell}{\sigma}$ can be viewed as the signal-to-noise ratio (SNR) of the employee's observed performance (cf. Bolton and Harris 1999, Bergemann and Valimaki 2000); we interpret it as the rate of learning 
about the employee's capabilities. In other words, we will say that low (high) $\sigma$ corresponds to a faster (slower) learning rate.

We assume that both the firm and the employee are risk neutral, with a common discount factor $\alpha<1$. For the duration of the employment, the employee earns a fixed wage $s$ and a proportion $\lambda \geq 0$ of the profit $X_{t}$ that the employee generates. Next, we specify the outside options for the firm and the employee. If the employment relationship is terminated, the firm will obtain its outside option $u$, which can include the expected net present value (NPV) of hiring another employee from the general pool of workers. On the other hand, the employee will obtain the outside option given by a random variable $W$, which represents the present value of the employee's lifetime income stream. The random variable $W$ depends on the employee's true capability: $W$ is equal to $w_{h}$ or $w_{\ell}$, depending on whether $\mu=h$ or $\mu=\ell$; naturally, $w_{h}>w_{\ell}$. This assumption is reasonable because a worker who is capable of contributing to a given firm's profit is likely to do the same for the profit of another firm in the same industry (see, e.g., Freeman 1977, Johnson 1978, Gonzalez and Shi 2010).

The payoffs for the players are the expected values of cumulative discounted profits. Let $E^{p}[\cdot] \equiv$ $E\left[\cdot \mid P_{0}=p\right]$ denote the expected value conditional on the initial belief $P_{0}=p$. The decision variable for each player is their time of separation. Let $\tau_{f}$ and $\tau_{e}$ be the discretionary time of separation for the firm and the employee, respectively. If we let $\tau_{S}=\tau_{f} \wedge \tau_{e}$ denote the time of separation, then the expected payoffs for the firm and the employee are, respectively,

$$
\begin{aligned}
V_{f}\left(p ; \tau_{S}\right) & =E^{p}\left[\int_{0}^{\tau_{S}}((1-\lambda) \mu-s) e^{-\alpha t} d t+\int_{0}^{\tau_{S}} \sigma e^{-\alpha t} d B_{t}+u e^{-\alpha \tau_{S}}\right] \\
& =\frac{1-\lambda}{\alpha} E^{p}[\mu]-\frac{s}{\alpha}+E^{p}\left[e^{-\alpha \tau_{S}} g_{f}\left(P_{\tau_{S}}\right)\right], \\
V_{e}\left(p ; \tau_{S}\right) & =E^{p}\left[\int_{0}^{\tau_{S}}(\lambda \mu+s) e^{-\alpha t} d t+W e^{-\alpha \tau_{S}}\right] \\
& =\frac{s}{\alpha}+\frac{\lambda}{\alpha} E^{p}[\mu]+E^{p}\left[e^{-\alpha \tau_{S}} g_{e}\left(P_{\tau_{S}}\right)\right] .
\end{aligned}
$$

Here we define

$$
g_{f}(p)=u-\frac{1-\lambda}{\alpha} E^{p}[\mu]+\frac{s}{\alpha}, \quad g_{e}(p)=E^{p}[W]-\frac{\lambda}{\alpha} E^{p}[\mu]-\frac{s}{\alpha} .
$$


These terms denote, respectively, the firm's and the employee's expected NPV of being in the employment relationship relative to the outside option given the belief that the employee is of high capability type with probability $p$. The payoff functions thus depend on $\tau_{S}$ through $E^{p}\left[e^{-\alpha \tau_{S}} g_{f}\left(P_{\tau_{S}}\right)\right]$ and $E^{p}\left[e^{-\alpha \tau_{S}} g_{e}\left(P_{\tau_{S}}\right)\right]$. The objective of the firm (employee) is to maximize its payoff by choosing the optimal time $\tau_{f}\left(\tau_{e}\right)$ of separation given the stopping time chosen by the employee (firm).

Now we will show that the problem above is equivalent to one with zero sharing of the profit in which some model parameters are transformed. We define $\alpha^{\prime}=\alpha /(1-\lambda), s^{\prime}=s /(1-\lambda), w_{h}^{\prime}=$ $w_{h}-\lambda h / \alpha, w_{\ell}^{\prime}=w_{\ell}-\lambda \ell / \alpha, \sigma^{\prime}=\sqrt{1-\lambda} \sigma$, a re-scaled time $t^{\prime}=t(1-\lambda)$, and $\tau_{S}^{\prime}=\tau_{S}(1-\lambda)$. By virtue of the property of a Brownian motion, $B_{t}=B_{t^{\prime} /(1-\lambda)}=\sqrt{1-\lambda} B_{t^{\prime}}$. Then the payoff functions can be re-expressed as

$$
\begin{aligned}
V_{f}(p ; S) & =E^{p}\left[\int_{0}^{\tau_{S}^{\prime}}\left(\mu-s^{\prime}\right) e^{-\alpha^{\prime} t^{\prime}} d t^{\prime}+\int_{0}^{\tau_{S}^{\prime}} \sigma^{\prime} e^{-\alpha^{\prime} t^{\prime}} d B_{t^{\prime}}+u e^{-\alpha^{\prime} \tau_{S}^{\prime}}\right] \\
& =\frac{1}{\alpha^{\prime}} E^{p}[\mu]-\frac{s^{\prime}}{\alpha^{\prime}}+E^{p}\left[e^{-\alpha^{\prime} \tau_{S}^{\prime}} \tilde{g}_{f}\left(P_{\tau_{S}^{\prime}}\right)\right] \\
V_{e}(p ; S) & =E^{p}\left[\int_{0}^{\tau_{S}^{\prime}}\left(\lambda \mu /(1-\lambda)+s^{\prime}\right) e^{-\alpha^{\prime} t^{\prime}} d t^{\prime}+W e^{-\alpha^{\prime} \tau_{S}^{\prime}}\right] \\
& =\frac{s^{\prime}}{\alpha^{\prime}}+\frac{\lambda}{\alpha(1-\lambda)} E^{p}[\mu]+E^{p}\left[e^{-\alpha^{\prime} \tau_{S}^{\prime}} \tilde{g}_{e}\left(P_{\tau_{S}^{\prime}}\right)\right]
\end{aligned}
$$

where

$$
\tilde{g}_{f}(p)=u-\frac{1}{\alpha^{\prime}} E^{p}[\mu]+\frac{s^{\prime}}{\alpha^{\prime}}, \quad \tilde{g}_{e}(p)=E^{p}\left[W^{\prime}\right]-\frac{s^{\prime}}{\alpha^{\prime}},
$$

with $W^{\prime}=w_{h}^{\prime}$ with probability $p$ and $W^{\prime}=w_{\ell}^{\prime}$ with probability $(1-p)$.

The objectives of the firm and the employee are to maximize $E^{p}\left[e^{-\alpha^{\prime} \tau_{S}^{\prime}} \tilde{g}_{f}\left(P_{\tau_{S}^{\prime}}\right)\right]$ and $E^{p}\left[e^{-\alpha^{\prime} \tau_{S}^{\prime}} \tilde{g}_{e}\left(P_{\tau_{S}^{\prime}}\right)\right]$, respectively. Thus, the equilibrium is solely determined by the functional forms of $\tilde{g}_{f}(\cdot)$ and $\tilde{g}_{e}(\cdot)$. Furthermore, the dependence on $\sigma$ lies solely in $E^{p}\left[e^{-\alpha^{\prime} \tau_{S}^{\prime}} \tilde{g}_{f}\left(P_{\tau_{S}^{\prime}}\right)\right]$ and $E^{p}\left[e^{-\alpha^{\prime} \tau_{S}^{\prime}} \tilde{g}_{e}\left(P_{\tau_{S}^{\prime}}\right)\right]$. Finally, we note that $\tilde{g}_{f}(\cdot)$ and $\tilde{g}_{e}(\cdot)$ are identical to $g_{f}(\cdot)$ and $g_{e}(\cdot)$ for $\lambda=0$ if we identify the primed parameters as non-primed parameters. Thus, the characteristics of equilibrium can be examined solely for cases of $\lambda=0$. Without loss of generality, we will therefore proceed with our exposition using the simplified form for $\lambda=0$. Moreover, we are interested in the 
regime of model parameters in which the firm wants to dismiss a low capability type employee and retain a high capability type employee, so we assume that $(h-s) / \alpha>u>(\ell-s) / \alpha$. Similarly, the employee will want to quit only if the employee is of a high capability type, and so we assume that $w_{h}>s / \alpha>w_{\ell}$.

\section{Benchmark Model: Cooperative Setting}

As a benchmark, we investigate a Nash bargaining solution between the firm and the employee (Papageorgiou 2013). Papageorgiou (2013) shows that through Nash bargaining, a cooperative separation decision is made along with a nontrivial salary structure that depends on the posterior belief. In this section, we analyze the cooperative separation decision of a similar Nash bargaining solution. In particular, we examine the comparative statics of the payoffs with respect to the learning rate.

\subsection{Cooperative Termination Time}

In the cooperative model, the firm and the employee agree on the termination time $\tau^{*}$ that maximizes their combined payoff, and they divide the payoff according to the Nash bargaining solution (Papageorgiou 2013). Note first that the expression for the total payoff is

$$
V_{c}(p ; \tau)=E^{p}\left[\int_{0}^{\tau} \mu e^{-\alpha t} d t+(u+W) e^{-\alpha \tau}\right]=\frac{E^{p}[\mu]}{\alpha}+E^{p}\left[g_{c}\left(P_{\tau}\right) e^{-\alpha \tau}\right],
$$

where $\tau$ is the agreed stopping time for separation and

$$
g_{c}(p)=u+E^{p}[W]-\frac{E^{p}[\mu]}{\alpha} .
$$

Note that $g_{c}(\cdot)$ is the sum of $g_{f}(\cdot)$ and $g_{e}(\cdot)$ because we are considering total payoff in the cooperative model. In the Nash bargaining solution, the disagreement points for the firm and the employee are their outside options, $u$ and $E^{p}[W]=p w_{h}+(1-p) w_{\ell}$, respectively. Thus, according to the canonical bargaining solution of Nash (1950), the respective payoffs for the employee $V_{e}(p)$ and the firm $V_{f}(p)$ are given by the following:

$$
\begin{aligned}
V_{e}(p ; \tau) & =\frac{1}{2}\left\{V_{c}(p ; \tau)-u-E^{p}[W]\right\}+E^{p}[W], \\
V_{f}(p ; \tau) & =\frac{1}{2}\left\{V_{c}(p ; \tau)-u-E^{p}[W]\right\}+u .
\end{aligned}
$$

The following lemma shows the structure of the optimal termination time. 
LEMma 1. If $w_{h}-w_{\ell}-h / \alpha+\ell / \alpha<0$, then there is a lower threshold $\theta_{d}$ such that $\tau^{*}=\inf \{t>0$ : $\left.P_{t}<\theta_{d}\right\}$ is the optimal stopping time that maximizes $V_{c}(p ; \tau)$. Similarly, if $w_{h}-w_{\ell}-h / \alpha+\ell / \alpha>0$, then there is an upper threshold $\theta_{u}$ such that $\tau^{*}=\inf \left\{t>0: P_{t}>\theta_{u}\right\}$ is the optimal stopping time that maximizes $V_{c}(p ; \tau)$.

The lemma states that in the cooperative model, it is optimal for the firm and employee to agree upfront on the employment separation terms, namely, that they will separate once the common posterior belief that the employee is a high capability type, $P_{t}$, falls below (rises above) an optimally chosen threshold $\theta_{d}\left(\theta_{u}\right)$. If $w_{h}-w_{\ell}-h / \alpha+\ell / \alpha<0$, then the profit stream $\mu$ dominates the payoff $W$ to the employee upon termination, so the employer's incentive is accentuated. In this case, the optimal cooperative decision is to terminate the employment when the employee is believed to be of low quality, so the optimal policy is to terminate it when $P_{t}$ falls below a threshold $\theta_{d}$. On the other hand, if $w_{h}-w_{\ell}-h / \alpha+\ell / \alpha>0$, then the employee's incentive is accentuated in the cooperative decision, so the optimal policy represents the employee's incentive, i.e., to terminate the employment when the employee is believed to be of high quality, so the optimal policy is to terminate it when $P_{t}$ climbs above a threshold $\theta_{u}$.

\subsection{Effect of Learning Rate}

The cooperative Nash bargaining solution shows that the problem basically amounts to a conventional optimal stopping (i.e., a real options) problem, and that the value function

$$
V_{c}(p)=\sup _{\tau>0}\left\{\frac{E^{p}[\mu]}{\alpha}+E^{p}\left[\left(g_{f}+g_{e}\right)\left(P_{\tau}\right) e^{-\alpha \tau}\right]\right\}=\frac{E^{p}[\mu]}{\alpha}+E^{p}\left[\left(g_{f}+g_{e}\right)\left(P_{\tau^{*}}\right) e^{-\alpha \tau^{*}}\right]
$$

is always nondecreasing with the speed of learning $1 / \sigma$. The comparative statics for payoffs from a central decision making problem with respect to the learning rate was obtained and analyzed in detail by Ryan and Lippman (2003). We formalize this result in the following proposition:

Proposition 1 (Benchmark). Under the cooperative setting, both of the value functions $V_{e}(p)$ and $V_{f}(p)$ are always non-increasing with $\sigma$. 
The intuition is very simple: for the combined problem, the faster learning is better for the centralized (cooperative) decision maker because the faster gain of information can be exploited to make a better decision earlier. Furthermore, we can specify the form of the payment transfer between the two parties as follows:

Proposition 2. The Nash bargaining solution is achieved if the per unit time wage paid to the employee is of the form

$$
s\left(P_{t}\right)=\frac{1}{2}\left\{E^{P_{t}}[\mu-\alpha W]+u\right\}=\frac{1}{2}\left\{P_{t}\left(h-\alpha w_{h}\right)+\left(1-P_{t}\right)\left(\ell-\alpha w_{\ell}\right)+u\right\} .
$$

\section{Noncooperative Model of a Separation Game}

We now investigate the equilibrium of the noncooperative game theoretic model. We will first characterize the structure of the best responses of the firm and the employee and show the existence of Markov perfect equilibrium (MPE) strategies and payoffs. We will then examine how these are impacted by the rate of learning and illustrate how this differs from the benchmark cooperative model.

\subsection{Markov Perfect Equilibrium}

In the noncooperative model, the employment separation time $\tau_{S}$ is the smaller of the firm's and the employee's stopping times $\tau_{f}$ and $\tau_{e}: \tau_{S}=\min \left\{\tau_{f}, \tau_{e}\right\}$. The firm's objective is therefore to find a $\tau_{f}$ that maximizes Eq. (1) given the employee's strategy $\tau_{e}$, and the employee's objective is to find a $\tau_{e}$ that maximizes Eq. (2) given the firm's strategy $\tau_{f}$.

When seeking the equilibrium strategies, we can restrict our attention to stationary Markov strategies because (a) the posterior process $P_{t}$ is a Markov process and (b) neither $g_{f}(\cdot)$ nor $g_{e}(\cdot)$ depends on calendar time (Oksendal 2003, p. 220). A stationary policy for player $i \in\{f, e\}$ can be represented by the state $P_{t}$ - specifically, by whether or not $P_{t} \in C_{i} \subset[0,1]$ for some open set $C_{i}$. In other words, the set $C_{i}$ denotes the stopping strategy for $\tau_{f}=\inf \left\{t \geq 0: P_{t} \notin C_{f}\right\}$ and $\tau_{e}=\inf \left\{t \geq 0: P_{t} \notin C_{e}\right\}$. We now show that these sets can be characterized in terms of thresholds with respect to $P_{t}$. 
Lemma 2. If there exists a best response $C_{f}^{*}$ to a given strategy $C_{e}$, then $C_{f}^{*}=\left(\theta_{f}, 1\right]$ for some $\theta_{f}$ that depends on $C_{e}$. Similarly, if there exists a best response $C_{e}^{*}$ to a given strategy $C_{f}$, then $C_{e}^{*}=\left[0, \theta_{e}\right)$ for some $\theta_{e}$ that depends on $C_{f}$.

This lemma is intuitive and straightforward. The firm does not want to terminate a high capability type employee, so it waits until the posterior $P_{t}$ is sufficiently low. Similarly, the employee will want to quit the job only if the employee is sufficiently optimistic about his or her capability; hence the employee waits until the posterior $P_{t}$ is high enough. Lemma 2 enables us to characterize the stopping times $\tau_{i}$ for $i \in\{f, e\}$ in terms of a pair of thresholds $\theta_{f}$ and $\theta_{e}$ :

$$
\tau_{f}=\inf \left\{t>0: P_{t} \leq \theta_{f}\right\}, \quad \tau_{e}=\inf \left\{t>0: P_{t} \geq \theta_{e}\right\}
$$

We will limit our attention to strategy profiles characterized by the pair of thresholds. If $\theta_{e}>\theta_{f}$, then the interval $\left(\theta_{f}, \theta_{e}\right)$ represents the region of continued employment. The expressions for each player's payoffs given the strategy profiles, i.e., $V_{f}\left(p ; \theta_{f}, \theta_{e}\right)$ and $V_{e}\left(p ; \theta_{f}, \theta_{e}\right)$, are provided in the Appendix (see Lemmas A-1 and A-2, respectively).

Next, we examine the strategic interaction between the firm and the employee. To understand their interaction, note that a higher threshold $\theta_{f}$ implies that the firm will initiate termination of the employee earlier. So from the employee's viewpoint, the firm's threat of termination is greater when $\theta_{f}$ is higher. Similarly, a lower $\theta_{e}$ implies that the employee will leave the firm sooner; so from the firm's viewpoint, the employee's threat of leaving is greater when $\theta_{e}$ is lower. The following proposition shows that a player's threat increases with the opponent's threat.

Proposition 3. (i) The employee's best response $\theta_{e}$ is non-increasing with $\theta_{f}$.

(ii) The firm's best response $\theta_{f}$ is non-increasing with $\theta_{e}$.

The rationale for these claims is as follows. If $\theta_{f}$ increases, then the employee is terminated earlier. An earlier termination decreases the overall expected payoff for the employee of remaining employed, which in turn induces the employee to quit (and seek his or her outside option) earlier and hence decreases $\theta_{e}$. Likewise, if $\theta_{e}$ decreases, then the employee quits earlier, which decreases 
the firm's overall expected payoff from the employment relationship. This decrease in turn induces the firm to dismiss the employee and seek its outside option earlier, thus increasing $\theta_{f}$.

An equilibrium strategy profile $\left(\theta_{f}^{*}, \theta_{e}^{*}\right)$ is one where $\theta_{f}^{*}$ and $\theta_{e}^{*}$ are the best responses to each other. Note that an MPE can include an equilibrium strategy profile $\left(\theta_{f}^{*}, \theta_{e}^{*}\right)$ that leads to immediate termination of employment, i.e., $\theta_{f}^{*} \geq \theta_{e}^{*}$; we call this a degenerate profile. For example, the degenerate strategy profile $\left(\theta_{f}, \theta_{e}\right)$ with $\theta_{f}=1$ and $\theta_{e}=0$ is always an MPE. The following assumption provides a sufficient condition for the existence of a non-degenerate MPE (i.e., $\theta_{f}^{*}<\theta_{e}^{*}$ ), which is where our interest lies.

Assumption 1. $\left(\frac{s-w_{\ell} \alpha}{w_{h} \alpha-s}\right)\left(\frac{h-s-u \alpha}{u \alpha+s-\ell}\right)>1$.

In essence, this assumption indicates when it is in the interest of both the firm and the employee to continue their employment relationship. To see this, note first that $s-w_{\ell} \alpha$ corresponds to the employee's loss from leaving if the employee has low capability, while $w_{h} \alpha-s$ corresponds to the employee's gain from leaving if the employee has high capability. Hence a larger value of $\left(s-w_{\ell} \alpha\right) /\left(w_{h} \alpha-s\right)$ signifies a greater loss than the gain from leaving, which induces the employee to leave later (i.e., the threshold $\theta_{e}$ is higher). Conversely, $h-s-u \alpha$ corresponds to the firm's loss when dismissing a high capability employee, while $u \alpha+s-\ell$ corresponds to its gain when dismissing a low capability employee. Hence a larger $(h-s-u \alpha) /(u \alpha+s-\ell)$ ratio incentivizes the firm to dismiss the employee later, i.e., the threshold $\theta_{f}$ is lower. We now obtain the MPE strategies for our model.

Proposition 4. Under Assumption 1, there exists an MPE with a strategy profile $\left(\theta_{f}^{*}, \theta_{e}^{*}\right)$ that satisfies $\theta_{f}^{*}<\theta_{e}^{*}$. Furthermore,

(i) a unique Pareto-dominant MPE is characterized by the highest ratio $\theta_{e}^{*} / \theta_{f}^{*}$ among all MPEs;

(ii) for sufficiently small $\sigma$, there exists a unique non-degenerate MPE.

Although the uniqueness of an MPE cannot be guaranteed in general, there always exists a unique Pareto-dominant MPE, according to Proposition 3(i). Furthermore, based on extensive 
numerical study, we conjecture that the non-degenerate MPE is unique if Assumption 1 is satisfied. In particular, for a sufficiently small $\sigma$, we can prove that the non-degenerate MPE is unique. For the rest of the paper, we therefore restrict our attention to the unique Pareto-dominant MPE.

\subsection{Effects of the Learning Rate}

We now investigate how the rate of learning affects MPE strategies and payoffs. In particular, we examine the comparative statics of $\theta_{f}^{*}, \theta_{e}^{*}$, and the MPE payoffs $V_{f}^{*}(p) \equiv V_{f}\left(p ; \theta_{f}^{*}, \theta_{e}^{*}\right)$ and $V_{e}^{*}(p) \equiv$ $V_{e}\left(p ; \theta_{f}^{*}, \theta_{e}^{*}\right)$ with respect to $\sigma$. To derive analytical results and insights about MPE strategies and payoffs, we will first examine the comparative statics for limiting values of the volatility $\sigma$ (i.e., $\sigma \rightarrow 0$ and $\sigma \rightarrow \infty)$. While analytical results are intractable for the general values of $\sigma$, one can always compute the MPE strategies and payoffs numerically. We complement our analytical results by numerically illustrating $V_{f}^{*}(p)$ and $V_{e}^{*}(p)$ with respect to the volatility $\sigma$ for intermediate values of $\sigma$.

The following proposition analytically characterizes the small-/large- $\sigma$ behaviors of MPE strategies and payoffs.

Proposition 5. Given Assumption 1, the following statements hold:

(i) In the limit $\sigma \rightarrow 0$, we have $\theta_{e}^{*} \uparrow \theta_{e}^{0}<1$ and $\theta_{f}^{*} \downarrow \theta_{f}^{0}>0$. Moreover, for sufficiently small values of $\sigma$, the MPE payoffs $V_{f}^{*}(p)$ and $V_{e}^{*}(p)$ increase with $\sigma, \forall p \in\left(\theta_{f}^{0}, \theta_{e}^{0}\right)$.

(ii) In the limit $\sigma \rightarrow \infty$, we have $\theta_{e}^{*} \downarrow \theta_{e}^{\infty} \equiv\left(s / \alpha-w_{\ell}\right) /\left(w_{h}-w_{\ell}\right)$ and $\theta_{f}^{*} \uparrow \theta_{f}^{\infty} \equiv(u \alpha+s-\ell) /(h-\ell)$. Moreover, letting $\hat{p} \equiv \sqrt{\theta_{e}^{\infty} \theta_{f}^{\infty}} /\left(\sqrt{\theta_{e}^{\infty} \theta_{f}^{\infty}}+\sqrt{\left(1-\theta_{e}^{\infty}\right)\left(1-\theta_{f}^{\infty}\right)}\right)$, we have $V_{e}^{*}(p) \uparrow s / \alpha$ and $V_{f}^{*}(p) \downarrow$ $\left[E^{p}(\mu)-s\right] / \alpha, \forall p \in\left(\theta_{f}^{\infty}, \hat{p}\right)$, and $V_{e}^{*}(p) \downarrow s / \alpha$ and $V_{f}^{*}(p) \uparrow\left[E^{p}(\mu)-s\right] / \alpha, \forall p \in\left(\hat{p}, \theta_{e}^{\infty}\right)$.

Part (i) of Proposition 5 examines the region of fast learning rates (or small $\sigma$ ). The volatility $\sigma$ is low when the outcomes of an employee's tasks are relatively predictable (e.g., sales in known markets). In such settings, both the firm and the employee learn quickly about the latter's capability. Here, the MPE thresholds do not converge to extreme values (0 or 1$)$, i.e., $\theta_{e}^{*} \uparrow \theta_{e}^{0} \neq 1$ and $\theta_{f}^{*} \downarrow \theta_{f}^{0} \neq 0$, even as $\sigma \rightarrow 0$. This is due to the noncooperative interaction between the firm and the employee - namely, the mutual threat of employment termination (Proposition 3) reduces both 
players' expected payoffs (the values of waiting before termination), inducing both to terminate earlier.

Remarkably, in this region of high learning rates, the expected payoffs for both the firm and the employee increase with $\sigma$ (i.e., lowering the learning rate benefits both parties) as long as the prior probability $p$ is within an intermediate regime. This result, which is in stark contrast to that for the cooperative benchmark model of Section 3, is a direct consequence of the noncooperative interaction between the firm and the employee. When the employee's capability is uncertain, increased volatility in the employee's performance will delay the separation decisions of both the firm and the employee. Although neither party knows a priori which party will benefit the most from prolonged employment under such uncertainty, there is minimal downside risk because each party has the option of terminating the relationship at any point. More volatility in performance prolongs the employment relationship, which could prove to be favorable for both parties.

Part (ii) of Proposition 5 examines the region of slow learning rates (or large $\sigma$ ). The volatility $\sigma$ is high when the outcomes of the employee's tasks are relatively unpredictable (as in R\&D or new product sales). Under this condition, both the firm and the employee will learn very slowly about the employee's capability. Note that both players continue the employment relationship if $P_{t} \in\left(\theta_{f}^{\infty}, \theta_{e}^{\infty}\right)$, even in the limit as $\sigma \rightarrow \infty$. If $\sigma$ is very large, then the rate of learning is extremely low and convergence of $P_{t}$ to either threshold will take a long time. Hence, the decisions of both players are made as if their beliefs will never be updated. It follows that the players will either terminate the employment almost immediately or wait a long time before taking any action. The limiting values of the payoff functions $V_{e}^{*}(p) \rightarrow s / \alpha$ and $V_{f}^{*}(p) \rightarrow\left(E^{p}[\mu]-s\right) / \alpha$ follow as a result of discounting.

We observe that in the region of low learning rates, as $\sigma$ increases, the expected payoffs for the firm and the employee move in opposite directions. For higher values of $p$, with $p \in\left(\hat{p}, \theta_{e}^{\infty}\right)$, $V_{f}^{*}(p)$ increases with $\sigma$ while $V_{e}^{*}(p)$ decreases with $\sigma$. Slower learning benefits the firm because it delays the (likely capable) employee's decision to quit while it hurts the employee because it 


\begin{tabular}{|c||c|c|c|c|c|c|c|}
\hline & $\sigma \backslash p$ & $p \in\left(0, \theta_{f}^{0}\right)$ & $p \in\left(\theta_{f}^{0}, \theta_{f}^{\infty}\right]$ & $p \in\left[\theta_{f}^{\infty}, \hat{p}\right]$ & $p \in\left[\hat{p}, \theta_{e}^{\infty}\right]$ & $p \in\left[\theta_{e}^{\infty}, \theta_{e}^{0}\right)$ & $p \in\left(\theta_{e}^{0}, 1\right)$ \\
\hline \multirow{2}{*}{ Cooperative } & $\forall \sigma$ & $\frac{\partial V_{f}^{*}(p)}{\partial \sigma}<0$ & $\frac{\partial V_{f}^{*}(p)}{\partial \sigma}<0$ & $\frac{\partial V_{f}^{*}(p)}{\partial \sigma}<0$ & $\frac{\partial V_{f}^{*}(p)}{\partial \sigma}<0$ & $\frac{\partial V_{f}^{*}(p)}{\partial \sigma}<0$ & $\frac{\partial V_{f}^{*}(p)}{\partial \sigma}<0$ \\
$\frac{\partial V_{e}^{*}(p)}{\partial \sigma}<0$ & $\frac{\partial V_{e}^{*}(p)}{\partial \sigma}<0$ & $\frac{\partial V_{e}^{*}(p)}{\partial \sigma}<0$ & $\frac{\partial V_{e}^{*}(p)}{\partial \sigma}<0$ & $\frac{\partial V_{e}^{*}(p)}{\partial \sigma}<0$ & $\frac{\partial V_{e}^{*}(p)}{\partial \sigma}<0$ \\
\hline \hline \multirow{2}{*}{ Noncooperative } & N/A & $\frac{\partial V_{f}^{*}(p)}{\partial \sigma}>0$ & $\frac{\partial V_{f}^{*}(p)}{\partial \sigma}>0$ & $\frac{\partial V_{f}^{*}(p)}{\partial \sigma}>0$ & $\frac{\partial V_{f}^{*}(p)}{\partial \sigma}>0$ & N/A \\
\cline { 2 - 7 } & low $\sigma$ & $\frac{\partial V_{e}^{*}(p)}{\partial \sigma}>0$ & $\frac{\partial V_{e}^{*}(p)}{\partial \sigma}>0$ & $\frac{\partial V_{e}^{*}(p)}{\partial \sigma}>0$ & $\frac{\partial V_{e}^{*}(p)}{\partial \sigma}>0$ & N/A \\
\cline { 2 - 7 } & N/A & N/A & $\frac{\partial V_{f}^{*}(p)}{\partial \sigma}<0$ & $\frac{\partial V_{f}^{*}(p)}{\partial \sigma}>0$ & N/A & N/A \\
high $\sigma$ & N/A & $\frac{\partial V_{e}^{*}(p)}{\partial \sigma}>0$ & $\frac{\partial V_{e}^{*}(p)}{\partial \sigma}<0$ & N/A & N/A \\
\hline
\end{tabular}

Table 1 Summary of comparative statics of $V_{f}^{*}(p)$ and $V_{e}^{*}(p)$ for different regions of $\sigma$ and $p$.

delays the employee's desired confirmation of being highly capable. For lower values of $p$, with $p \in\left(\theta_{f}^{\infty}, \hat{p}\right], V_{f}^{*}(p)$ decreases with $\sigma$ while $V_{e}^{*}(p)$ increases with $\sigma$. In this case, slower learning hurts the firm because it delays the confirmation of the (likely incapable) employee's low capability while it benefits the employee because it delays the firm's decision to terminate employment. It is interesting to note that while a lower learning rate no longer benefits both parties, it continues to benefit at least one party; this differs from the cooperative benchmark case, in which slower learning never benefits any party.

Overall, the comparative statics of the payoffs for the firm and the employee have a rich structure with respect to the learning rate, and they exhibit stark differences between the cooperative model and the noncooperative model. The comparative statics results are summarized in Table 1.

Although it is not possible to analytically characterize the effect of the learning rate for the intermediate values of $\sigma$, one can numerically compute the equilibrium payoffs. To better understand these regions of $\sigma$, we have conducted extensive numerical studies. Figure 1 plots an illustrative representation of $V_{f}^{*}(p)$ and $V_{e}^{*}(p)$ with respect to $\sigma$ (top half) and with respect to $p$ (bottom half), and exhibits two key features. First, and most importantly, we observe that the comparative statics for the low $\sigma$-region (Proposition $4(i)$ ) holds as long as $\sigma<3$, and that for the high $\sigma$-region, (Proposition 4(ii)) holds as long as $\sigma>10$. In other words, the comparative statics for the limiting 
values of $\sigma$ described in Proposition 4 apply to intermediate values of $\sigma$. Second, we observe a relatively smooth crossover from the comparative statics results for the low $\sigma$-region to those for the high $\sigma$-region. In sum, the numerical analysis reveals that Proposition 4 applies to nontrivial regions of $\sigma$, and it provides important insights regarding the comparative statics for intermediate regions of $\sigma$.

Lastly, we remark that it is not always true that slow learning prolongs the employer-employee relationship in our model. The relationship between the speed of learning and the time to termination depends on the value of the posterior as shown by Kwon and Lippman (2011). A slower learning has two countervailing effects on the time to termination. On one hand, slower learning may encourage the players to take more time to gather more information before making the termination decision. On the other hand, slower learning decreases the value of learning per unit time which may discourage the players from waiting longer to learn about the quality of the employee. Depending on which effect dominates, the slower learning may hasten or delay the termination decisions. For example, if $p$ takes an intermediate value within $\left(\theta_{f}^{0}, \theta_{e}^{0}\right)$, slower learning tends to delay the time to termination for sufficiently small values of $\sigma$, which leads to Proposition 5(i). In other regions of $p$, this may no longer be true, in which case the value functions do not necessarily increase with $\sigma$.

\section{Extensions and Robustness of the Main Results}

In this section, we check the robustness of our key results against the posterior-dependence of the wage (Section 5.1) and the potential improvement of the employee's capability through on-the-job learning (Section 5.2).

\subsection{Posterior-Dependent Wage}

Until now, we have assumed that the firm pays the employee a fixed salary. In this subsection, we relax that assumption and allow the firm to pay the employee more if the employee is believed to be capable. 

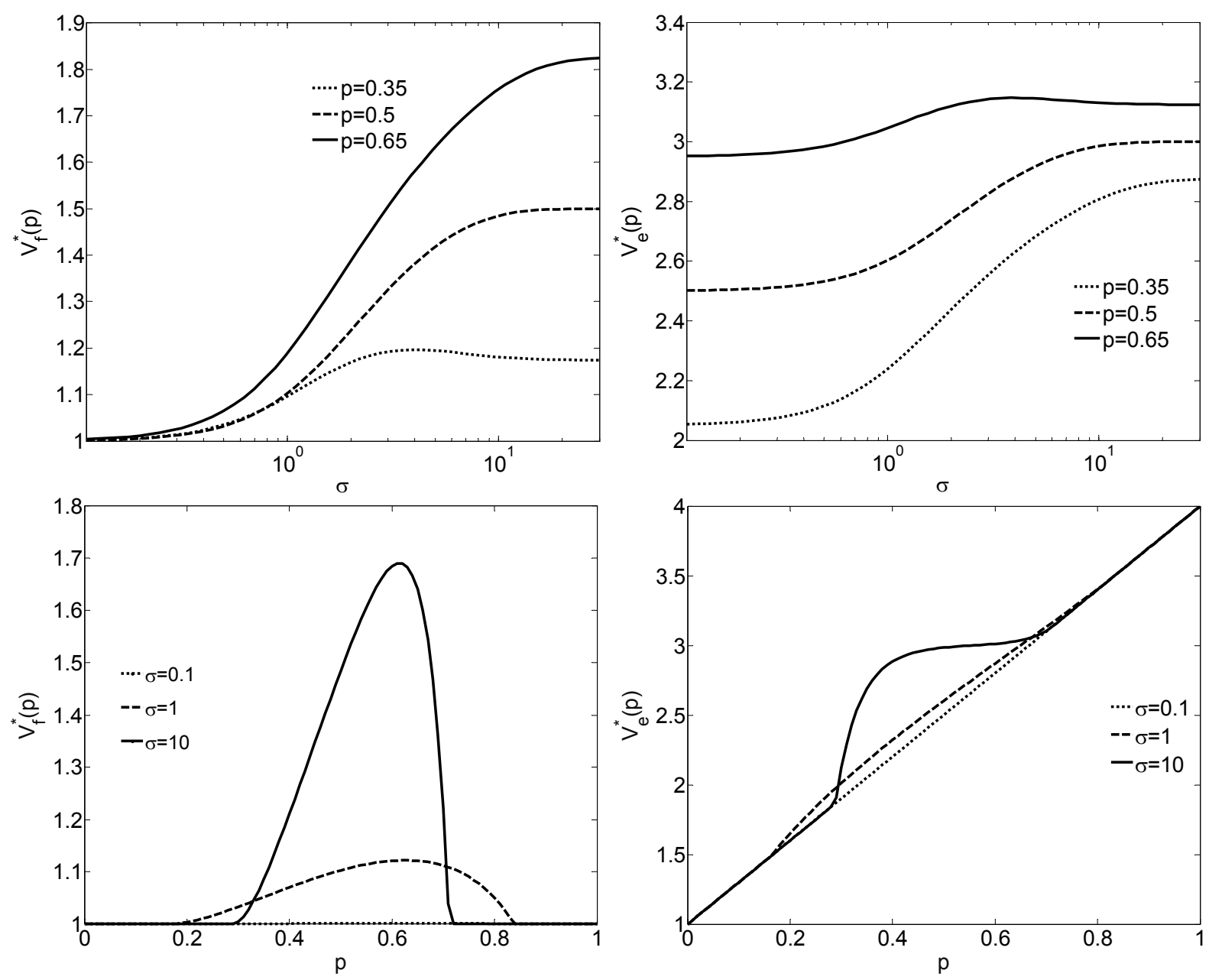

Figure $1 V_{f}^{*}(p)$ and $V_{e}^{*}(p)$. (Parameters: $\alpha=1, h=6, \ell=3, s=3, w_{h}=4, w_{\ell}=1$, and $u=1 ; \theta_{f}^{0}=0.07, \theta_{e}^{0}=0.93$, $\theta_{f}^{\infty}=0.33, \theta_{e}^{\infty}=0.67, \hat{p}=0.5$.)

Linear wage contract. First, we consider the simplest possible posterior- dependence of the wage: a wage schedule that depends linearly on the posterior $p$. Suppose that the wage is given by $s(p)=s_{0}+s_{1} p$ for some $s_{1}>0$. Then the payoffs are given by the following:

$$
\begin{aligned}
& V_{f}\left(p ; \tau_{S}\right)=E^{p}\left[\int_{0}^{\tau_{S}}\left(\mu-s\left(P_{t}\right)\right) e^{-\alpha t} d t+u e^{-\alpha \tau_{S}}\right]=\frac{1}{\alpha}\left[E^{p}[\mu]-s(p)\right]+E^{p}\left[e^{-\alpha \tau_{S}} g_{f}\left(P_{\tau_{S}}\right)\right], \\
& V_{e}\left(p ; \tau_{S}\right)=E^{p}\left[\int_{0}^{\tau_{S}} s\left(P_{t}\right) e^{-\alpha t} d t+W e^{-\alpha \tau_{S}}\right]=\frac{1}{\alpha} s(p)+E^{p}\left[e^{-\alpha \tau_{S}} g_{e}\left(P_{\tau_{S}}\right)\right],
\end{aligned}
$$

where

$$
g_{f}(p)=u-\frac{1}{\alpha}\left\{E^{p}[\mu]-s(p)\right\}, \quad g_{e}(p)=E^{p}[W]-\frac{s(p)}{\alpha} .
$$


The payoffs thus depend on $\tau_{S}$ through $E^{p}\left[e^{-\alpha \tau_{S}} g_{f}\left(P_{\tau_{S}}\right)\right]$ and $E^{p}\left[e^{-\alpha \tau_{S}} g_{e}\left(P_{\tau_{S}}\right)\right]$. Note that if we replace $h-s_{1}$ with $h$ and $w_{h}-s_{1} / \alpha$ with $w_{h}$, then the forms of $g_{f}(\cdot)$ and $g_{e}(\cdot)$ reduce to the forms with $s_{1}=0$. Hence, as long as $h-s_{1}-\ell>0$ and $w_{h}-s_{1} / \alpha-w_{\ell}>0$, the main results of the paper continue to apply to this case. If $h-s_{1}-\ell<0$, then the wage schedule is too steeply in favor of the employee, so the best response of the firm is to dismiss the employee when $P_{t}$ is sufficiently high. Similarly, if $w_{h}-s_{1} / \alpha-w_{\ell}>0$, then the wage schedule is so much in favor of the firm that the employee's best response is to leave the firm only if $P_{t}$ is sufficiently low. Overall, a wage that increases with $P_{t}$ does not affect the main results, provided that $s_{1}$ is not unreasonably high.

We hasten to add that our result is robust to a profit-sharing compensation plans as given by (1) and (2). In fact, we can map any profit-sharing plan to one with no profit-sharing compensation using exactly the same argument presented at the end of Section 2. However, in case of a nonlinear wage, i.e., if $s(\cdot)$ is non-linear, then the mapping no longer holds true, and the profit-sharing compensation needs to be explicitly considered in addition to the wage.

Lastly, we remark that the wage structure (4) that achieves the Nash bargaining solution of Section 3.2 is also linear in posterior. However, the analysis of the current section does not apply to the Nash bargaining framework because the employer and the employee must cooperatively work together to achieve the bargaining solution whereas the present analysis applies to a noncooperative game.

Nonlinear wage contract. More complicated nonlinear posterior-dependent wages can be incorporated in the model in a similar manner, although general analytical expressions may not be available. One particular nonlinear form of wage contract that turns out to yield a particularly tractable form of the solution is a logarithmic wage of the form $s(x)=s_{0}+s_{1} \ln \frac{x}{1-x}$. Using the same parameter values as Figure 1, Figure 2 plots $V_{e}^{*}(p)$ and $V_{f}^{*}(p)$ assuming this logarithmic wage function, with $s_{0}=3$ and $s_{1}=0.2$ with respect to $\sigma$ (top half) and with respect to $p$ (bottom half). Comparison of Figure 2 with Figure 1 reveals that the main results (Proposition 4) of the paper continue to hold even in the case of a nonlinear wage schedule, namely, both $V_{e}^{*}(p)$ and $V_{f}^{*}(p)$ increase with $\sigma$ for smaller values of $\sigma$, and either $V_{f}^{*}(p)$ or $V_{e}^{*}(p)$ increases with $\sigma$ for larger values of $\sigma$. 

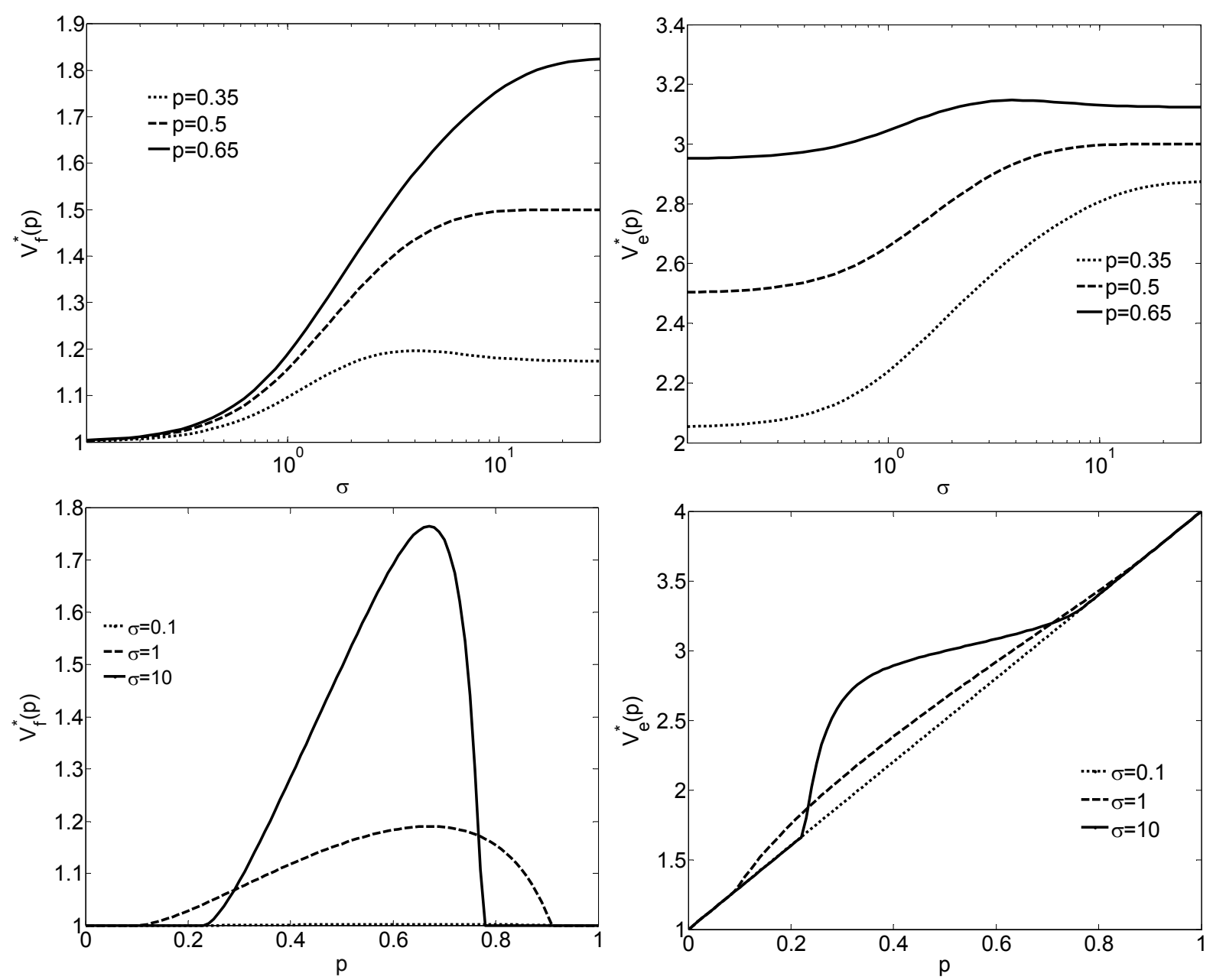

Figure $2 V_{f}^{*}(p)$ and $V_{e}^{*}(p)$ under a nonlinear wage contract $s(p)=3+0.2 \ln \frac{p}{1-p}$. (Parameters: $\alpha=1, h=6, \ell=3$, $s=3, w_{h}=4, w_{\ell}=1$, and $\left.u=1.\right)$

\subsection{Impact of On-the-job Learning}

So far, we have assumed that a low quality employee remains low quality throughout the duration of the employment relationship. In this subsection, we relax this assumption and allow a low quality employee to become a high quality one through on-the-job learning.

We assume that the transformation takes place at a random exponential time with an arrival rate of $\eta>0$. For notational convenience, we define $\bar{\mu}=(h+\ell) / 2$ and closely follow the formulation provided by Ryan and Lippman (2005). By virtue of Ryan and Lippman (2005), the posterior probability $P_{t}=P\left(\{\mu=h\} \mid \mathcal{F}_{t}\right)$ that a given employee is of high quality can be expressed as

$$
P_{t}=\frac{(1-p) \int_{0}^{t} \exp \left\{\frac{\beta}{\sigma}\left[X_{t}-X_{s}-\bar{\mu}(t-s)\right]\right\} \eta e^{-\eta s} d s+p \exp \left[\frac{\beta}{\sigma}\left(X_{t}-\bar{\mu} t\right)\right]}{(1-p) e^{-\eta t}+(1-p) \int_{0}^{t} \exp \left\{\frac{\beta}{\sigma}\left[X_{t}-X_{s}-\bar{\mu}(t-s)\right]\right\} \eta e^{-\eta s} d s+p \exp \left[\frac{\beta}{\sigma}\left(X_{t}-\bar{\mu} t\right)\right]},
$$



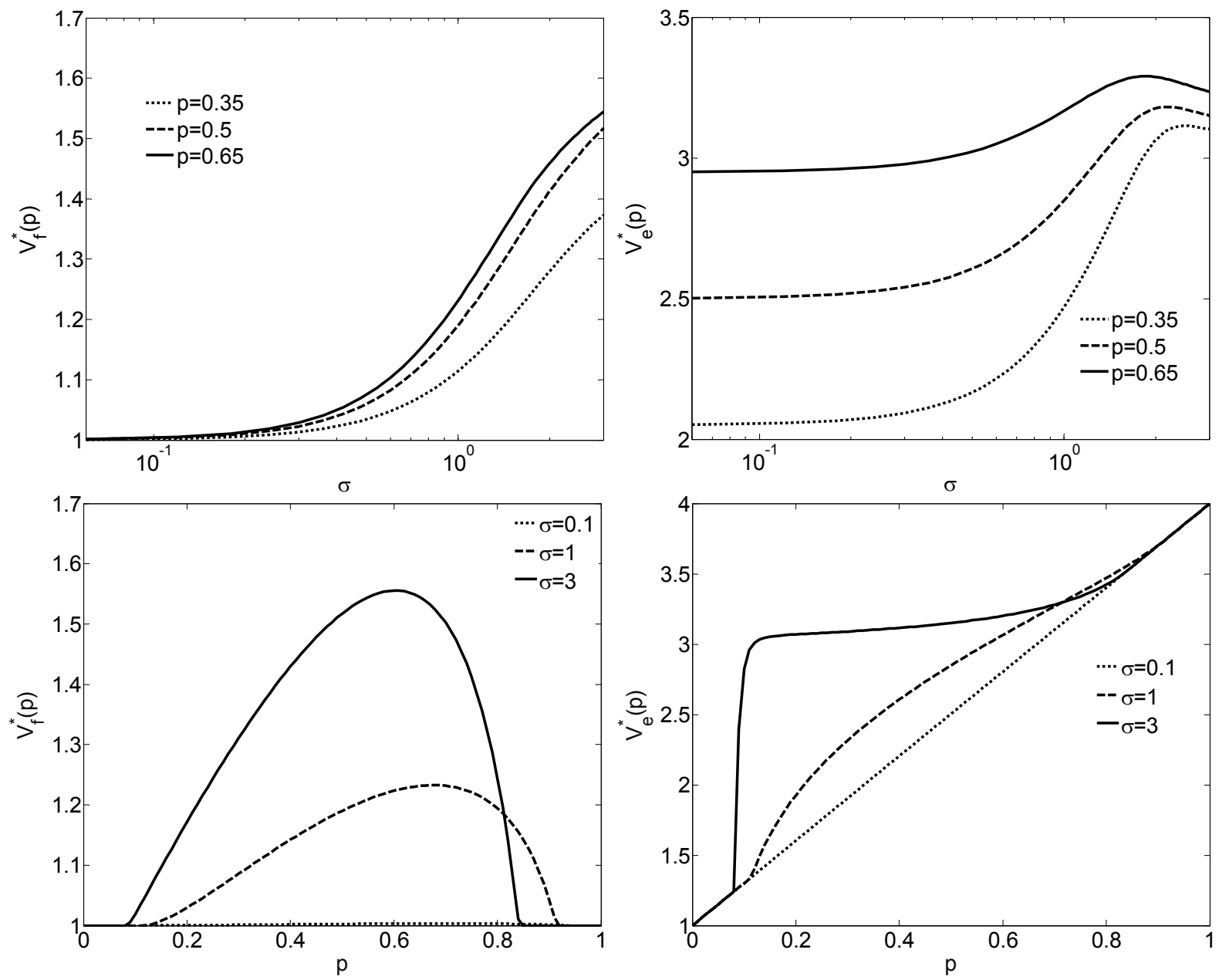

Figure $3 V_{f}^{*}(p)$ and $V_{e}^{*}(p)$ under on-the-job learning with rate $\eta=0.5$. (Parameters: $\alpha=1, h=6, \ell=3, s=3$, $\left.w_{h}=4, w_{\ell}=1, u=1.\right)$

where $p=P_{0}$ is the initial posterior. The SDE of $P_{t}$ is given by

$$
d P_{t}=\eta\left(1-P_{t}\right) d t+\beta P_{t}\left(1-P_{t}\right) d \tilde{W}_{t}
$$

where

$$
\tilde{W}_{t}:=\frac{1}{\sigma}\left\{X_{t}-\int_{0}^{t}\left(P_{s} h+\left(1-P_{s}\right) \ell\right) d s\right\}
$$

The MPE strategies and the associated payoff functions can be derived using the same formalism utilized in Kwon (2013). Using the same model parameter values as in Figure 1, Figure 3 plots $V_{e}^{*}(p)$ and $V_{f}^{*}(p)$ with arrival rate $\eta=0.5$ with respect to $\sigma$ (top half) and with respect to $p$ (bottom half). Comparison with Figure 1 demonstrates that the main results of the paper (Proposition 4) 
continue to hold once again, namely, both $V_{e}^{*}(p)$ and $V_{f}^{*}(p)$ increase with $\sigma$ for smaller values of $\sigma$, and at least one party benefits from slower learning (i.e., either $V_{f}^{*}(p)$ or $V_{e}^{*}(p)$ increases with $\sigma)$ for larger values of $\sigma$.

\section{Discussion}

In this section, we discuss the implications of our research, which are twofold. First, from a theoretical perspective, one contribution is the discovery of an atypical example of a game in which a faster learning rate (more information per unit time) can decrease the payoff for both players. More specifically, we find that in the region of high learning rates (low $\sigma$ ), the equilibrium for both players, $V_{f}^{*}(p)$ and $V_{e}^{*}(p)$, can increase with $\sigma$. This is precisely the opposite of the conventional wisdom that a higher learning rate is generally beneficial, as was also shown in the cooperative benchmark case.

Second, our result points to an active nonfinancial strategy that firms can employ to increase their retention of capable employees: Firms can control the learning rate $\sigma$ by assigning their new employees to tasks in which their capabilities will be revealed more quickly or more slowly. For example, a consulting firm may provide its new engagement managers with incentives/penalties for seeking new clients in new sectors, and an entrepreneurial firm might encourage its VP to develop and market products that inherently rely more on luck (i.e., that are more challenging) or discourage the VP from doing so. If luck plays a relatively large (small) role in performance outcomes, employees will be criticized less (more) for failing to generate revenue, and they will also take less (more) credit for their short-term successes. This approach to retaining employees is robust to change in compensation packages or on-the-job learning by employees.

Specifically, according to Table 1, the firm should first examine whether the new employee is assigned to tasks with values of $\sigma$ that are too low. If so, it behooves the firm to encourage the employee to take more risks in order to increase $\sigma$, because doing so mutually benefits both parties. This is because this will prolong the duration of employment while each party retains the option of terminating employment. If the task is relatively volatile, i.e., the value of $\sigma$ is high, then firms 
should next examine the prevailing values of $p$. If both the firm and the employee think that the employee is likely to be capable $(p>\hat{p})$, then it behooves the firm to encourage the employee to take on more volatile projects, as doing so benefits the firm but hurts the employee. On the other hand, if $p<\hat{p}$, then the firm should reverse that approach.

\section{Conclusions}

This paper examined the setting in which an employee is hired into a new role and both the firm and the employee must learn about the employee's capability. This learning process presents both parties with an option: The firm can terminate the employee's employment if the employee turns out not to be capable, and the employee can leave the firm for greater financial remuneration if he or she turns out to be highly capable. We explored how this noncooperative interaction impacts the duration of the relationship and which party (if any) benefits from a faster learning rate. Our analysis provides counterintuitive and practical insights into how firms can manage noncooperative interactions and retain capable employees.

\section{References}

Alvarez, L. H. R. 2003. On the properties of r-excessive mappings for a class of diffusions. Annals of Applied Probability 13 1517-1533.

Arlotto, A., S. E. Chick, N. Gans. 2014. Optimal hiring and retention policies for heterogeneous workers who learn. Management Science 60(1) 110-129.

Aumann, R. J. 1976. Agreeing to disagree. The Annals of Statistics 4(6) 1236-1239.

Bassamboo, A., J. M. Harrison, A. Zeevi. 2006. Design and control of a large call center: Asymptotic analysis of an lp-based method. Operations Research 54(3) 419-435.

Bergemann, D., J. Valimaki. 2000. Experimentation in markets. The Review of Economic Studies 67(2) $213-234$.

Bitran, G. R., E. A. Haas, A. C. Hax. 1981. Hierarchical production planning: A single stage system. Operations Research 29(4) 717-743.

Bolton, P., C. Harris. 1999. Strategic experimentation. Econometrica 67(2) 349-374.

Decamps, J.-P., T. Mariotti, S. Villeneuve. 2005. Investment timing under incomplete information. Mathematics of Operations Research 30(2) 472-500. 
Dixit, A. 1989. Entry and exit decisions under uncertainty. The Journal of Political Economy 97(3) 620-638.

Dixit, A. 1992. Investment and hysteresis. The Journal of Economic Perspectives 6(1) 107-132.

Freeman, S. 1977. Wage trends as performance displays productive potential: A model and application to academic early retirement. The Bell Journal of Economics 8(2) pp. 419-443.

Gans, N., Y.-P. Zhou. 2002. Managing learning and turnover in employee staffing. Operations Research 50(6) 991-1006.

Gonzalez, F. M., S. Shi. 2010. An equilibrium theory of learning, search, and wages. Econometrica 78(2) $509-537$.

Gurvich, I., M. Lariviere, A. Moreno. 2015. Operations in the on-demand economy: Staffing services with self-scheduling capacity. Working Paper .

Holt, C., F. Modigliani, J. Muth, H. Simon. 1960. Planning Production, Inventories, and Workforce. PrenticeHall, Englewood Cliffs, NJ.

Johnson, W. R. 1978. A theory of job shopping. The Quarterly Journal of Economics 92(2) pp. 261-278.

Jovanovic, B. 1979. Job matching and the theory of turnover. The Journal of Political Economy 87(5) pp. 972-990.

Kreps, D. M. 1988. Notes On The Theory Of Choice. Westview Press, Boulder, Boulder, Colorado.

Kwon, H. D. 2010. Invest or Exit? Optimal Decisions in the Face of a Declining Profit Stream. Operations Research 58(3) 638-649.

Kwon, H. D. 2013. Prevention of catastrophic failures with weak forewarning signals. Probability in the Engineering and Informational Sciences 28(1) 121-144.

Kwon, H. D., S. A. Lippman. 2011. Acquisition of project-specific assets with bayesian updating. Operations Research 59(5) 1119-1130.

Liptser, R., A. Shiryayev. 1977. Statistics of Random Processes I. Springer-Verlag, Berlin.

Nash, J. F. 1950. The bargaining problem. Econometrica 18(2) 155-162.

Oksendal, B. 2003. Stochastic Differential Equations: An Introduction with Applications. 6th ed. Springer, Berlin.

Papageorgiou, T. 2013. Learning your comparative advantages. The Review of Economic Studies .

Peskir, G., A. Shiryaev. 2006. Optimal Stopping and Free-Boundary Problems. Birkhauser Verlag, Basel.

Pinker, E. J., R. A. Shumsky. 2000. The efficiency-quality trade-off of cross-trained workers. Manufacturing Eamp; Service Operations Management 2(1) 32-48. 
Ryan, R., S. A. Lippman. 2003. Optimal exit from a project with noisy returns. Probab. Engrg. Inform. Sci. $\mathbf{1 7}(04) 435-458$.

Ryan, R., S. A. Lippman. 2005. Optimal exit from a deteriorating project with noisy returns. Probability in the Engineering and Informational Sciences 19(03) 327-343.

Shiryaev, A. N. 1967. Two problems of sequential analysis. Cybernetics and Systems Analysis 3(2) 63-69.

Viscusi, W. K. 1980. A theory of job shopping: A bayesian perspective. The Quarterly Journal of Economics 94(3) pp. 609-614.

\section{Appendix. Mathematical Proofs.}

Proofs of Lemma 1 and Proposition 1. See Ryan and Lippman (2003).

Proof of Proposition 2. If $s\left(P_{t}\right)$ is the wage paid to the employee, then the employee's payoff is given by

$$
U_{e}(p)=E^{p}\left[\int_{o}^{\tau^{*}} s\left(P_{t}\right) e^{-\alpha t} d t+W e^{-\alpha \tau^{*}}\right],
$$

which must equal to $V_{e}\left(p ; \tau^{*}\right)$ given by (3). Theory of stopping problems (Oksendal 2003) dictates that $\mathcal{A} U_{e}(p)=-s(p)$ must be satisfied, where $\mathcal{A}$ is the characteristic differential operator for $P_{t}$ (Peskir and Shiryaev 2006) given by

$$
\mathcal{A} \equiv-\alpha+\frac{1}{2}\left(\frac{h-\ell}{\sigma}\right)^{2} p^{2}(1-p)^{2} \partial_{p}^{2}
$$

Hence, $s(p)=-\mathcal{A} V_{e}\left(p ; \tau^{*}\right)$, which leads to (4).

Proof of Lemma 2. In order to obtain the best response to the opponent's strategy, we need to utilize optimal stopping theory. The most direct way to find the optimal solution is to construct a candidate value function $V_{i}\left(p ; C_{f}, C_{e}\right)$ which is a return function to a candidate policy $C_{i}$, and to verify that it satisfies a number of sufficient conditions as laid out by Theorem 10.4.1 of Oksendal (2003). One of the conditions stipulates that $\mathcal{A} V_{i}\left(p ; C_{f}, C_{e}\right)=0$. Here the term $-\alpha$ replaces the term $\partial_{t}$ from the time-dependent characteristic operator (Oksendal 2003) because the payoff from Markov strategies is time-invariant except for the discount factor $e^{-\alpha t}$. The positive fundamental solutions to the equation $\mathcal{A} f(p)=0$ are given by equations (5). Note that $\phi(\cdot)$ is convex decreasing while $\psi(\cdot)$ is convex increasing. Then the value function $V_{i}\left(p ; C_{f}, C_{e}\right)$ is given by a linear combination of $\phi(\cdot)$ and $\psi(\cdot)$.

We first consider the firm's best response. We note that $\mathcal{A} g_{f}(p)=-\alpha g_{f}(p)$. Because $g_{f}(\cdot)$ is a decreasing function and because of the inequalities $(h-s) / \alpha>u>(\ell-s) / \alpha$, there is $y \in(0,1)$ such that $\mathcal{A} g_{f}(p)>0$ for $p>y$ and $\mathcal{A} g_{f}(p)<0$ for $p<y$. By the argument of Oksendal (2003), p. 215, the best response of the firm must contain $(y, 1]$ and cannot have a component disconnected from $(y, 1]$. Thus, the best response is $\left(\theta_{f}, 1\right]$ for some $\theta_{f}<y$ which depends on $C_{e}$. Using an analogous argument, we can show that the employee's best response is $\left[0, \theta_{e}\right)$ for some $\theta_{e}$ which depends on $C_{f}$.

The next two lemmas establish each player's unique best response and expected payoff given the other player's strategy, and will be useful for Proposition. Let $\gamma \equiv \sqrt{1+\left(8 \alpha \sigma^{2}\right) /(h-\ell)^{2}}$ and

$$
\phi(p) \equiv p^{(1-\gamma) / 2}(1-p)^{(1+\gamma) / 2}, \quad \psi(p) \equiv p^{(1+\gamma) / 2}(1-p)^{(1-\gamma) / 2} .
$$


Lemma A-1. The employee's best response is determined as follows:

(i) If $g_{e}\left(\theta_{f}\right) \geq 0$, then the employee's best response is to quit immediately $\left(\theta_{e} \leq \theta_{f}\right)$, and $V_{e}\left(p ; \theta_{f}, \theta_{e}\right)=$ $s / \alpha+g_{e}(p)$ for all $p \in[0,1]$.

(ii) If $g_{e}\left(\theta_{f}\right)<0$, then there exists a unique best response $\theta_{e}>\theta_{f}$ that satisfies the equality $a_{1} \phi\left(\theta_{f}\right)+$ $a_{2} \psi\left(\theta_{f}\right)=g_{e}\left(\theta_{f}\right)$, where

$$
a_{1}=\frac{\psi\left(\theta_{e}\right)}{2 \gamma}\left[\left(w_{h}-\frac{s}{\alpha}\right) \frac{\gamma-1}{1-\theta_{e}}+\left(w_{\ell}-\frac{s}{\alpha}\right) \frac{\gamma+1}{\theta_{e}}\right], a_{2}=\frac{\phi\left(\theta_{e}\right)}{2 \gamma}\left[\left(w_{h}-\frac{s}{\alpha}\right) \frac{\gamma+1}{1-\theta_{e}}+\left(w_{\ell}-\frac{s}{\alpha}\right) \frac{\gamma-1}{\theta_{e}}\right] .
$$

The employee's expected payoff is

$$
V_{e}\left(p ; \theta_{f}, \theta_{e}\right)= \begin{cases}\frac{s}{\alpha}+a_{1} \phi(p)+a_{2} \psi(p) & \text { for } p \in\left(\theta_{f}, \theta_{e}\right), \\ \frac{s}{\alpha}+g_{e}(p) & \text { otherwise. }\end{cases}
$$

LEMmA A-2. The firm's best response is determined as follows: $(i)$ If $g_{f}\left(\theta_{e}\right) \geq 0$, then the firm's best response is to dismiss the employee immediately $\left(\theta_{f} \geq \theta_{e}\right)$ and $V_{f}\left(p ; \theta_{f}, \theta_{e}\right)=\frac{1}{\alpha}[p h+(1-p) \ell-s]+g_{f}(p)$ for all $p \in[0,1]$.

(ii) If $g_{f}\left(\theta_{e}\right)<0$, then there exists a unique best response $\theta_{f}<\theta_{e}$ that satisfies the equality $b_{1} \phi\left(\theta_{e}\right)+$ $b_{2} \psi\left(\theta_{e}\right)=g_{f}\left(\theta_{e}\right)$, where

$b_{1}=\frac{\psi\left(\theta_{f}\right)}{2 \gamma}\left[\left(u+\frac{s-h}{\alpha}\right) \frac{\gamma-1}{1-\theta_{f}}+\left(u+\frac{s-\ell}{\alpha}\right) \frac{\gamma+1}{\theta_{f}}\right], \quad b_{2}=\frac{\phi\left(\theta_{f}\right)}{2 \gamma}\left[\left(u+\frac{s-h}{\alpha}\right) \frac{\gamma+1}{1-\theta_{f}}+\left(u+\frac{s-\ell}{\alpha}\right) \frac{\gamma-1}{\theta_{f}}\right]$.

The firm's expected payoff is

$$
V_{f}\left(p ; \theta_{f}, \theta_{e}\right)= \begin{cases}\frac{1}{\alpha}[p h+(1-p) \ell-s]+b_{1} \phi(p)+b_{2} \psi(p) & \text { for } p \in\left(\theta_{f}, \theta_{e}\right), \\ \frac{1}{\alpha}[p h+(1-p) \ell-s]+g_{f}(p) & \text { otherwise. }\end{cases}
$$

Proof of Lemma A-1. To prove this Proposition, we solve the optimal stopping time problem of the employee to obtain $\sup _{\tau_{e}} E^{p}\left[e^{-\alpha \tau_{e} \wedge \tau_{f}} g_{e}\left(P_{\tau_{e} \wedge \tau_{f}}\right)\right]$. Let's assume that $\theta_{f}<\theta_{e}$. Under the firm's strategy $\theta_{f}$, the domain of the employee's value function is restricted to $\left[\theta_{f}, 1\right]$. To prove the existence of the best response, we only need to find $\theta_{e}$ and the solution $f(\cdot)$ to $\mathcal{A} f(p)=0$ for $p \in\left(\theta_{f}, \theta_{e}\right)$ which is continuous in $\left[\theta_{f}, 1\right]$ and which satisfies the smooth-pasting condition $f^{\prime}\left(\theta_{e}\right)=g_{e}^{\prime}\left(\theta_{e}\right)$. The solution $f(\cdot)$ also has to satisfy $f(p) \geq g_{e}(p)$ for all $p \in\left[\theta_{f}, 1\right], f\left(\theta_{f}\right)=g_{e}\left(\theta_{f}\right)$, and $\mathcal{A} f(p) \leq 0$ for all $p \in\left(\theta_{e}, 1\right]$. In the end, $f(p)$ is identified

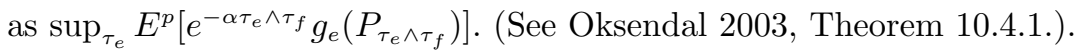

From $\mathcal{A} f(p)=0$ for $p \in\left(\theta_{f}, \theta_{e}\right)$ and the continuity, $f(\cdot)$ must have the following form:

$$
f(p)= \begin{cases}a_{1} \phi(p)+a_{2} \psi(p) & \text { for } p \in\left[\theta_{f}, \theta_{e}\right] \\ g_{e}(p) & \text { for } p \in\left\{\theta_{f}\right\} \cup\left[\theta_{e}, 1\right]\end{cases}
$$

for some coefficients $a_{1}$ and $a_{2}$. The coefficients $a_{1}$ and $a_{2}$ are determined by the conditions $f\left(\theta_{e}\right)=g_{e}\left(\theta_{e}\right)$ and $f^{\prime}\left(\theta_{e}\right)=g_{e}^{\prime}\left(\theta_{e}\right)$. 
We first establish that a unique solution $\theta_{e}$ to

$$
a_{1} \phi\left(\theta_{f}\right)+a_{2} \psi\left(\theta_{f}\right)=g_{e}\left(\theta_{f}\right)
$$

exists if $g_{e}\left(\theta_{f}\right)<0$. Equation (A4) is derived from the condition $f\left(\theta_{f}\right)=g_{e}\left(\theta_{f}\right)$. Define $\beta_{f} \equiv \theta_{f} /\left(1-\theta_{f}\right)$, $\beta_{e} \equiv \theta_{e} /\left(1-\theta_{e}\right)$, and $\eta \equiv \beta_{e} / \beta_{f}$. Note that $\beta_{f}\left(\beta_{e}\right)$ is strictly increasing in $\theta_{f}\left(\theta_{e}\right)$ and that $\eta>1$ if $\theta_{f}<\theta_{e}$. From Eq. (A4) we obtain the following equation for $\eta$ :

$$
\begin{gathered}
\frac{s / \alpha-w_{\ell}}{\left(w_{h}-s / \alpha\right) \beta_{f}}=\frac{j(\eta, \gamma)}{j\left(\eta^{-1}, \gamma\right)} \\
\text { where } \quad j(\eta, \gamma)=\eta^{(\gamma+1) / 2}(\gamma-1)+\eta^{-(\gamma-1) / 2}(\gamma+1)-2 \gamma .
\end{gathered}
$$

It is straightforward to prove that $j(\eta, \gamma)$ takes a minimum value of 0 at $\eta=1$ and is strictly positive for $\eta \neq 1$. Since we are interested in $\eta>1, j(\eta, \gamma)$ and $j\left(\eta^{-1}, \gamma\right)$ are strictly positive. Let

$$
m(\eta, \gamma) \equiv \frac{j(\eta, \gamma)}{j\left(\eta^{-1}, \gamma\right)}
$$

After some algebra, we obtain

$$
\partial_{\eta} m(\eta, \gamma)=\frac{\left(\gamma^{2}-1\right)}{\left[j\left(\eta^{-1}, \gamma\right)\right]^{2} \eta}\left(\eta^{\gamma / 2}-\eta^{-\gamma / 2}\right)\left[\eta^{\gamma / 2}-\eta^{-\gamma / 2}-\gamma\left(\eta^{1 / 2}-\eta^{-1 / 2}\right)\right] .
$$

It is straightforward to prove that $\eta^{\gamma / 2}-\eta^{-\gamma / 2}-\gamma\left(\eta^{1 / 2}-\eta^{-1 / 2}\right)>0$ for all $\eta>1$ and $\gamma>1$ because its derivative with respect to $\eta$ is strictly positive for $\eta>1$. It follows that $\partial_{\eta} m(\eta, \gamma)>0$ for all $\eta>1$ and $\gamma>1$. We also note that $j(\eta, \gamma) / j\left(\eta^{-1}, \gamma\right) \rightarrow 1$ in the limit $\eta \downarrow 1$ and $j(\eta, \gamma) / j\left(\eta^{-1}, \gamma\right) \rightarrow \infty$ in the limit $\eta \rightarrow \infty$. Hence, $j(\eta, \gamma) / j\left(\eta^{-1}, \gamma\right)$ monotonically increases in $\eta$ if $\eta>1$, and it can take any value in $(1, \infty)$. We conclude that there is a unique value of $\eta \in(1, \infty)$ which satisfies Eq. (A5) given $\beta_{f}, w_{h}, w_{\ell}$, and $s / \alpha$ as long as $\frac{s / \alpha-w_{\ell}}{\left(w_{h}-s / \alpha\right) \beta_{f}}>1$, which is equivalent to the condition $g_{e}\left(\theta_{f}\right)<0$.

We observe that $\theta_{f}<\theta_{e}$ can be satisfied only if $g_{e}\left(\theta_{f}\right)<0$. If $g_{e}\left(\theta_{f}\right) \geq 0$, then there is no solution $\eta>1$ that satisfies Eq. (A5). Thus, the employee's best response is to quit immediately. This proves statement (ii).

Next, we prove $f(p) \geq g_{e}(p)$. We first inspect the sign of $a_{1}$ and $a_{2}$. Suppose that both $a_{1}$ and $a_{2}$ have the same sign, either positive or negative. Since $\phi(\cdot)$ and $\psi(\cdot)$ are both convex, $f(p)=a_{1} \phi(p)+a_{2} \psi(p)$ is either strictly convex or strictly concave, and it cannot intersect with a linear function $g_{e}(p)$ twice (at $\theta_{e}$ and $\theta_{f}$ ) if $f^{\prime}\left(\theta_{e}\right)=g_{e}^{\prime}\left(\theta_{e}\right)$ is satisfied. Hence, $a_{1}$ and $a_{2}$ must have opposite signs. If $a_{1}>0$ and $a_{2}<0$, then $f(\cdot)$ is monotonically decreasing, which contradicts the condition $f\left(\theta_{f}\right)=g_{e}\left(\theta_{f}\right)<g_{e}\left(\theta_{e}\right)=f\left(\theta_{e}\right)$. Thus, $a_{1}<0$ and $a_{2}>0$.

Since $f(\cdot)$ cannot be strictly convex or concave in the interval $\left[\theta_{f}, \theta_{e}\right], f(\cdot)$ must be concave-convex from the functional form of $\phi(\cdot)$ and $\psi(\cdot)$, i.e., $f(p)$ must be concave for $p<p_{I}$ and convex for $p>p_{I}$ for some inflection point $p_{I} \in\left(\theta_{f}, \theta_{e}\right)$. It follows that $f(p)-g_{e}(p)$ is concave-convex which vanishes at $p \in\left\{\theta_{f}, \theta_{e}\right\}$ with a vanishing first derivative at $p=\theta_{e}$. The only way this is possible is if $f^{\prime}(p)-g_{e}^{\prime}(p)$ is positive at $\theta_{f}$, turns negative once somewhere in the interval $\left(\theta_{f}, \theta_{e}\right)$, and approaches zero as $p \rightarrow \theta_{e}$. It follows that $f(p) \geq g_{e}(p)$ for all $p \in\left[\theta_{f}, 1\right]$.

Now we confirm the inequality $\mathcal{A} f(p) \leq 0$ for $p \in\left(\theta_{f}, 1\right]$. Because $\mathcal{A} f(p)=0$ for $p \in\left(\theta_{f}, \theta_{e}\right)$, we only need to check the interval $\left[\theta_{e}, 1\right]$. From $\mathcal{A} f(p)=0$ and $\partial_{p}^{2} f(p)>0$ for $p \uparrow \theta_{e}$, we find that $f\left(\theta_{e}\right)>0$ or $g_{e}\left(\theta_{e}\right)>0$. That implies that $\mathcal{A} g_{e}(p)=-\alpha g_{e}(p)<0$ for $p>\theta_{e}$ since $g_{e}(\cdot)$ is increasing. This proves that the solution $\theta_{e}$ to Eq. (A4) is the best response threshold. 
Proof of Lemma A-2. Proof is analogous to Proof of Lemma A-1.

Proof of Proposition 3. Suppose that the given threshold $\theta_{f}$ of the firm increases. This implies that the firm will dismiss the employee earlier even if the employee would like to stay. Let $\theta_{f}^{\prime}>\theta_{f}$ and $\tau_{f}=\inf \{t \geq$ $\left.0: P_{t} \leq \theta_{f}\right\}, \tau_{f}^{\prime}=\inf \left\{t \geq 0: P_{t} \leq \theta_{f}^{\prime}\right\}$. Because $\tau_{f} \geq \tau_{f}^{\prime}$, the employee's optimal expected gain from stopping satisfies the following:

$$
\sup _{\tau \geq 0} E^{p}\left[e^{-\alpha \tau \wedge \tau_{f}} g_{e}\left(P_{\tau \wedge \tau_{f}}\right)\right]=\sup _{0 \leq \tau \leq \tau_{f}} E^{p}\left[e^{-\alpha \tau} g_{e}\left(P_{\tau}\right)\right] \geq \sup _{0 \leq \tau \leq \tau_{f}^{\prime}} E^{p}\left[e^{-\alpha \tau} g_{e}\left(P_{\tau}\right)\right]=\sup _{\tau \geq 0} E^{p}\left[e^{-\alpha \tau \wedge \tau_{f}^{\prime}} g_{e}\left(P_{\tau \wedge \tau_{f}^{\prime}}\right)\right]
$$

Hence, the payoff to the employee decreases in $\theta_{f}$. If the payoff $f(p)$ to the employee decreases, then the best response upper threshold $\theta_{e}$ decreases because $\left(\theta_{f}, \theta_{e}\right)$ is identified as $\left\{p: f(p)>g_{e}(p)\right\}$. This proves that $\theta_{e}$ decreases in $\theta_{f}$. We can use a similar argument to prove that $\theta_{f}$ also decreases in $\theta_{e}$.

Proof of Proposition 4. (i) Suppose $\theta_{e}$ and $\theta_{f}$ are best responses to each other. Then they must satisfy Eq. (A5). Similarly, from Proposition 2,

$$
b_{1} \phi\left(\theta_{e}\right)+b_{2} \psi\left(\theta_{e}\right)=g_{f}\left(\theta_{e}\right)
$$

which is the condition that $\theta_{f}$ is the best response to $\theta_{e}$, the following condition has to be satisfied:

$$
\left(\frac{h-s-u \alpha}{u \alpha+s-\ell}\right) \beta_{e}=\frac{j(\eta, \gamma)}{j\left(\eta^{-1}, \gamma\right)},
$$

where $j(\eta, \gamma)$ is given by Eq. (A6).

The thresholds $\theta_{e}$ and $\theta_{f}$ are completely determined if and only if $\beta_{e}=\theta_{e} /\left(1-\theta_{e}\right)$ and $\beta_{f}=\theta_{f} /\left(1-\theta_{f}\right)$ are determined. Hence, it suffices to determine the values of $\beta_{e} \beta_{f}$ and $\eta \equiv \beta_{e} / \beta_{f}$. From the ratio of Eqs. (A5) to $(\mathrm{A} 8)$, the value of $\beta_{e} \beta_{f}$ is given by

$$
\left(\frac{s-w_{\ell} \alpha}{w_{h} \alpha-s}\right)\left(\frac{u \alpha+s-\ell}{h-s-u \alpha}\right)=\beta_{e} \beta_{f} .
$$

There is a value of $\beta_{e} \beta_{f} \in(0, \infty)$ which satisfies this equation because the left-hand-side is positive. Next, by multiplying Eqs. (A5) and (A8), we obtain

$$
\left(\frac{s-w_{\ell} \alpha}{w_{h} \alpha-s}\right)\left(\frac{h-s-u \alpha}{u \alpha+s-\ell}\right)=k(\eta, \gamma)
$$

where the right-hand-side

$$
k(\eta, \gamma) \equiv \frac{1}{\eta}\left[\frac{j(\eta, \gamma)}{j\left(\eta^{-1}, \gamma\right)}\right]^{2}
$$

takes the value of 1 in the limit $\eta \downarrow 1$ and $\infty$ in the limit $\eta \rightarrow \infty$. Hence, there exists at least one value of $\eta>1$ which satisfies this equation as long as the left-hand-side is larger than 1.

Note: Assumption 1 is actually a necessary and sufficient condition for an MPE with $\theta_{f}<\theta_{e}$ if the function $k(\eta, \gamma)$ is strictly larger than 1 for all $\eta>1$ and $\gamma>1$. Our numerical study indicates that $k(\eta, \gamma)$ is larger than 1 for all values of $\eta \in(1,100)$ and $\gamma \in(1,100)$, so we speculate that Assumption 1 is a necessary and sufficient condition for an MPE with $\theta_{f}<\theta_{e}$. Moreover, the same numerical study shows that $k(\eta, \gamma)$ is a strictly increasing function of $\eta$. From the apparent monotonicity of $k(\cdot, \gamma)$, we further speculate that there is a unique value of $\eta$ that satisfies Eq. (A10) and that the MPE is unique.

Next, note that there are a finite number of values of $\eta$ which satisfy Eq. (A10) because the function $k(\eta, \gamma)$ is continuously differentiable and $k(\eta, \gamma) \rightarrow \infty$ in the limit $\eta \rightarrow \infty$. Thus, there are a finite number 
of MPEs. Let $n$ be the total number of MPEs, and let $S_{i}=\left(\theta_{f, i}, \theta_{e, i}\right)$ denote the $i$ th MPE strategy profile with the firm's threshold $\theta_{f, i}$ and the employee's threshold $\theta_{e, i}$. In particular, we index $S_{i}$ in such a way that $\theta_{e, i}>\theta_{e, j}$ if $i<j$. Then $\theta_{f, 1}<\theta_{f, 2}$ must be true because the firm's best response $\theta_{f}$ must decrease in the strategy $\theta_{e}$ of the employee by Lemmas A-1 and A-2. Hence, $\theta_{f, 1}<\theta_{f, 2}<\theta_{e, 2}<\theta_{e, 1}$ must be satisfied. From the proof of Lemma A-1, we note that $V_{e}\left(p ; \theta_{f, 2}, \theta_{e, 2}\right) \leq V_{e}\left(p ; \theta_{f, 1}, \theta_{e, 2}\right)$ because $\theta_{f, 2}>\theta_{f, 1}$. By the property of MPEs, we have

$$
V_{e}\left(p ; S_{2}\right)=V_{e}\left(p ; \theta_{f, 2}, \theta_{e, 2}\right) \leq V_{e}\left(p ; \theta_{f, 1}, \theta_{e, 2}\right) \leq V_{e}\left(p ; \theta_{f, 1}, \theta_{e, 1}\right)=V_{e}\left(p ; S_{1}\right) .
$$

Similarly, we can show that $V_{f}\left(p ; S_{2}\right) \leq V_{f}\left(p ; S_{1}\right)$. We can repeat the same argument for all $i$ between 2 and $n$ and conclude that $V_{e}\left(p ; S_{1}\right) \geq V_{e}\left(p ; S_{i}\right)$ and $V_{f}\left(p ; S_{1}\right) \geq V_{f}\left(p ; S_{i}\right)$. Thus, $S_{1}$, which has the highest ratio $\theta_{e, i} / \theta_{f, i}$, is the Pareto-dominant MPE.

(ii) Since $\gamma \downarrow 1$ as $\sigma \rightarrow 0$, it suffices to study the limits of small values of $\delta \equiv \gamma-1$. We note that

$$
j(\eta, 1+\delta)=(x-1-\ln x) \delta+\frac{1}{2}(\ln x)\left(x-1+\frac{1}{2} \ln x\right) \delta^{2}+O\left(\delta^{3}\right)
$$

in the limit $\delta \rightarrow 0$. Hence, Eq. (A10) reduces to

$$
\left(\frac{s-w_{\ell} \alpha}{w_{h} \alpha-s}\right)\left(\frac{h-s-u \alpha}{u \alpha+s-\ell}\right)=\frac{(\eta-1-\ln \eta)^{2}}{\eta\left(\eta^{-1}-1+\ln \eta\right)^{2}}+O(\delta) .
$$

Let

$$
k(\eta) \equiv \frac{(\eta-1-\ln \eta)^{2}}{\eta\left(\eta^{-1}-1+\ln \eta\right)^{2}} .
$$

Now we prove that there is a unique MPE in the small- $\sigma$ limit by showing that $k(\eta)$ is strictly increasing for $\eta>1$. We note that

$$
\frac{d k(\eta)}{d \eta}=\frac{(\eta-1-\ln \eta)}{(1-\eta+\eta \ln \eta)^{3}} k_{1}(\eta)
$$

where $k_{1}(\eta) \equiv \eta(\ln \eta)^{2}+\left(\eta^{2}-1\right) \ln \eta-3 \eta^{2}+6 \eta-3$. We note that $k_{1}(1)=0$ and that its first and second derivatives vanishes at $\eta=1$, and its third derivative is zero at $\eta=1$ but strictly positive for $\eta>1$. Consequently, $d^{2} k_{1} / d \eta^{2}, d k_{1} / d \eta$, and $k_{1}(\eta)$ are all strictly positive and increasing for $\eta>1$. It follows that $k(\eta)$ is strictly increasing for all $\eta>1$.

Next, we prove that $\theta_{e}^{*}$ decreases in $\sigma$ while $\theta_{f}^{*}$ increases in $\sigma$ by showing that $\eta^{*}$ decreases in $\sigma$ in the small- $\sigma$ limit. To do so, we simply need to show $\lim _{\gamma \rightarrow 1^{+}} \partial_{\gamma} m\left(\eta^{*}, \gamma\right)>0$ and use the expression

$$
\frac{d \eta^{*}}{d \gamma}=-\left(\frac{2 m\left(\eta^{*}, \gamma\right)}{\eta^{*}}\right) \frac{\partial_{\gamma} m\left(\eta^{*}, \gamma\right)}{\partial_{\eta} k\left(\eta^{*}, \gamma\right)}
$$

which is derived by applying the implicit function theorem on Eq. (A10), since we already know that $d k(\eta) / d \eta>0$. After some algebra,

$$
\lim _{\gamma \rightarrow 1^{+}} \partial_{\gamma} m(\eta, \gamma)=\frac{(\eta-1-\ln \eta) \eta \ln \eta}{(1-\eta+\eta \ln \eta)^{3}} \cdot m_{1}(\eta),
$$

where

$$
m_{1}(\eta)=-2(\eta-1)^{2}+\frac{1}{2}\left(\eta^{2}-1\right) \ln \eta+\eta(\ln \eta)^{2} .
$$

We note that $m_{1}(1)=0$, and its first and second derivatives vanishes at $\eta=1$ and its third derivative is strictly positive for $\eta>1$ from the property of $\eta^{2}-2 \eta \ln \eta-1$. Consequently, $d^{2} m_{1} / d \eta^{2}, d m_{1} / d \eta$, and $m_{1}(\eta)$ are all increasing and strictly positive for $\eta>1$. It follows that $\lim _{\gamma \rightarrow 1^{+}} \partial_{\gamma} m(\eta, \gamma)>0$ for all $\eta>1$. Therefore, $d \eta^{*} / d \sigma<0$ is true, and $\theta_{e}^{*}$ decreases in $\sigma$ while $\theta_{f}^{*}$ increases in $\sigma$ from Eq. (A9). 
Proof of Proposition 5. (i) From Eq. (A11), we note that $\lim _{\eta \rightarrow 1} k(\eta)=1$ and $\lim _{\eta \rightarrow \infty} k(\eta)=\infty$. Hence, if $\left(\frac{s-w_{\ell} \alpha}{w_{h} \alpha-s}\right)\left(\frac{h-s-u \alpha}{u \alpha+s-\ell}\right)>1$, there exists a finite value of $\eta \in(1, \infty)$ which satisfies Eq. (A10) in the limit $\sigma \rightarrow 0$. It means that $\theta_{e}^{*} \nrightarrow \rightarrow 1$ and $\theta_{f}^{*} \nrightarrow \rightarrow 0$ in the limit $\sigma \rightarrow 0$.

Let $\theta_{f}^{*}(\sigma)$ and $\theta_{e}^{*}(\sigma)$ denote the MPE thresholds when the volatility is $\sigma$. Similarly, let $V_{e}^{*}(p ; \sigma)$ and $V_{f}^{*}(p ; \sigma)$ denote the MPE payoffs when the volatility is $\sigma$. For $p \in\left(\theta_{f}^{*}(\sigma), \theta_{e}^{*}(\sigma)\right)$, we study the limiting values $\lim _{\sigma \rightarrow 0} V_{e}^{*}(p ; \sigma)$ and $\lim _{\sigma \rightarrow 0} V_{f}^{*}(p ; \sigma)$. From Eq. (A2), $V_{e}^{*}(p ; \sigma)=s / \alpha+a_{1} \phi(p)+a_{2} \psi(p)$ where $a_{1}$ and $a_{2}$ are given in Proposition 1. For small values of $\sigma$,

$$
a_{1} \phi(p)+a_{2} \psi(p)=p w_{h}+(1-p) w_{\ell}-\frac{s}{\alpha}+O\left(\sigma^{2}\right)=g_{e}(p)+O\left(\sigma^{2}\right) .
$$

Hence, $\lim _{\sigma \rightarrow 0} V_{e}^{*}(p ; \sigma)=s / \alpha+g_{e}(p)$. However, $V_{e}^{*}(p ; \sigma)>s / \alpha+g_{e}(p)$ for $\sigma>0$ by the property of the best response of the employee. Thus, we conclude $V_{e}^{*}(p ; \sigma)>\lim _{\sigma \rightarrow 0} V_{e}^{*}(p ; \sigma)$. Using the same procedure, we can show that $V_{f}^{*}(p ; \sigma)>\lim _{\sigma \rightarrow 0} V_{f}^{*}(p ; \sigma)$.

From the fact that $a_{1}$ and $a_{2}$ have well-defined Taylor series with respect to $\sigma^{2}$, for any fixed value of $p$, we can express $V_{f}^{*}(p ; \sigma)=V_{f}^{*}(p ; 0)+C_{f}^{2 n}(p) \cdot \sigma^{2 n}+O\left(\sigma^{2 n+2}\right)$ and $V_{e}^{*}(p ; \sigma)=V_{e}^{*}(p ; 0)+C_{e}^{2 m}(p) \cdot \sigma^{2 m}+$ $O\left(\sigma^{2 m+2}\right)$ for some integers $n \geq 1$ and $m \geq 1$, where $C_{f}^{2 n}(p)$ and $C_{e}^{2 m}(p)$ are coefficients of the leading terms of Taylor expansions of $V_{f}^{*}$ and $V_{e}^{*}$. By virtue of the inequalities $V_{f}^{*}(p ; \sigma)>\lim _{\sigma \rightarrow 0} V_{f}^{*}(p ; \sigma)$ and $V_{e}^{*}(p ; \sigma)>$ $\lim _{\sigma \rightarrow 0} V_{e}^{*}(p ; \sigma)$, we conclude that these leading coefficients $C_{f}^{2 n}(p)$ and $C_{e}^{2 m}(p)$ are positive. Therefore, $V_{e}^{*}(p ; \sigma)$ and $V_{f}^{*}(p ; \sigma)$ increase with $\sigma$ for $p \in\left(\theta_{f}^{*}(\sigma), \theta_{e}^{*}(\sigma)\right)$ and for sufficiently small values of $\sigma$.

(ii) In the large- $\gamma$ limit, we find that

$$
m(\eta, \gamma)=\eta+O\left(\gamma^{-1}\right), \quad k(\eta, \gamma)=\eta+O\left(\gamma^{-1}\right) .
$$

From Eqs. (A8) and (A9), we find that $\beta_{e}^{*} \equiv \theta_{e}^{*} /\left(1-\theta_{e}^{*}\right)$ and $\beta_{f}^{*} \equiv \theta_{f}^{*} /\left(1-\theta_{f}^{*}\right)$ are given by

$$
\beta_{e}^{*}=\frac{s-w_{\ell} \alpha}{w_{h} \alpha-s}+O\left(\gamma^{-1}\right), \quad \beta_{f}^{*}=\frac{u \alpha+s-\ell}{h-s-u \alpha}+O\left(\gamma^{-1}\right) .
$$

So we obtain $\beta_{e}^{*} \rightarrow \beta_{e}^{\infty}, \beta_{f}^{*} \rightarrow \beta_{f}^{\infty}, \theta_{e}^{*} \rightarrow \theta_{e}^{\infty}$ and $\theta_{f}^{*} \rightarrow \theta_{f}^{\infty}$. The $O\left(\gamma^{-1}\right)$ terms can be obtained directly from Eqs. (A4) and (A7):

$$
\begin{aligned}
& \beta_{e}^{*}=\beta_{e}^{\infty}\left(\frac{\gamma+1}{\gamma-1}\right)+\frac{2 g_{e}\left(\theta_{f}^{\infty}\right) \sqrt{\beta_{e}^{\infty}}}{\left(w_{h}-s / \alpha\right) \sqrt{\theta_{f}^{\infty}\left(1-\theta_{f}^{\infty}\right)}}\left(\frac{\beta_{e}^{\infty}}{\beta_{f}^{\infty}}\right)^{-\gamma / 2}+o\left(\left(\beta_{e}^{\infty} / \beta_{f}^{\infty}\right)^{-\gamma / 2}\right), \\
& \beta_{f}^{*}=\beta_{f}^{\infty}\left(\frac{\gamma-1}{\gamma+1}\right)+\frac{2 g_{f}\left(\theta_{e}^{\infty}\right) \sqrt{\beta_{f}^{\infty}}}{(u+s / \alpha-h / \alpha) \sqrt{\theta_{e}^{\infty}\left(1-\theta_{e}^{\infty}\right)}}\left(\frac{\beta_{e}^{\infty}}{\beta_{f}^{\infty}}\right)^{-\gamma / 2}+o\left(\left(\beta_{e}^{\infty} / \beta_{f}^{\infty}\right)^{-\gamma / 2}\right) .
\end{aligned}
$$

Hence, $\theta_{e}^{*} \downarrow\left(s / \alpha-w_{\ell}\right) /\left(w_{h}-w_{\ell}\right)$ and $\theta_{f}^{*} \uparrow(u \alpha+s-\ell) /(h-\ell)$ as $\gamma \rightarrow \infty$.

To prove part (ii), we need to inspect the large- $\gamma$ behaviors of $a_{1} \phi(p)+a_{2} \psi(p)$ and $b_{1} \phi(p)+b_{2} \psi(p)$ [see Lemmas A-1 and A-2 for Eqs. (A2) and (A3)]. We insert Eq. (A12) into the expressions for $a_{1}$ and $a_{2}$ in Lemma A-1 to obtain the following:

$$
\begin{aligned}
& a_{1} \phi(p)=g_{e}\left(\theta_{f}^{\infty}\right) \sqrt{\frac{p(1-p)}{\theta_{f}^{\infty}\left(1-\theta_{f}^{\infty}\right)}}\left(\frac{p /(1-p)}{\beta_{f}^{\infty}}\right)^{-\gamma / 2}+o\left[\left(\frac{p /(1-p)}{\beta_{f}^{\infty}}\right)^{-\gamma / 2}\right], \\
& a_{2} \psi(p)=2\left(\frac{s}{\alpha}-w_{\ell}\right) \sqrt{\frac{p(1-p)}{\beta_{e}^{\infty}}} \frac{1}{\gamma}\left(\frac{\beta_{e}^{\infty}}{p /(1-p)}\right)^{-\gamma / 2}+o\left[\left(\frac{\beta_{e}^{\infty}}{p /(1-p)}\right)^{-\gamma / 2}\right] .
\end{aligned}
$$

Note that $\frac{p /(1-p)}{\beta_{f}^{\infty}}>1$ and $\frac{\beta_{e}^{\infty}}{p /(1-p)}>1$ because $\theta_{f}^{\infty}<p<\theta_{e}^{\infty}$. Both $a_{1} \phi(p)$ and $a_{2} \psi(p)$ converge to zero as $\gamma \rightarrow \infty$, but $a_{1} \phi(p)$ converges to zero more slowly than $a_{2} \psi(p)$ if and only if $p /(1-p) \leq \sqrt{\beta_{e}^{\infty} \beta_{f}^{\infty}}$. Hence, $a_{1} \phi(p)+a_{2} \psi(p) \uparrow 0$ for $p /(1-p) \leq \sqrt{\beta_{e}^{\infty} \beta_{f}^{\infty}}$ and $a_{1} \phi(p)+a_{2} \psi(p) \downarrow 0$ for $p /(1-p)>\sqrt{\beta_{e}^{\infty} \beta_{f}^{\infty}}$ because $a_{1} \phi(p)<$ $0<a_{2} \psi(p)$. Following an analogous procedure and using Eq. (A13), we can show that $b_{1} \phi(p)+b_{2} \psi(p) \uparrow 0$ for $p /(1-p) \geq \sqrt{\beta_{e}^{\infty} \beta_{f}^{\infty}}$ and $b_{1} \phi(p)+b_{2} \psi(p) \downarrow 0$ for $p /(1-p)<\sqrt{\beta_{e}^{\infty} \beta_{f}^{\infty}}$. 